IdeAs

Idées d'Amériques

$3 \mid 2012$

L'alimentation dans les Amériques au prisme des sciences sociales

\title{
La politique alimentaire et la consommation dans l'Argentine péroniste
}

Food Politics and Consumption in Peronist Argentina

La política alimentaria y el consumo en la Argentina Peronista

\section{Natalia MILANESIO}

\section{OpenEdition}

Journals

Édition électronique

URL : https://journals.openedition.org/ideas/425

DOI : $10.4000 /$ ideas.425

ISSN : 1950-5701

Éditeur

Institut des Amériques

\section{Référence électronique}

Natalia MILANESIO, « La politique alimentaire et la consommation dans I'Argentine péroniste », IdeAs [En ligne], 3 | 2012, mis en ligne le 13 décembre 2012, consulté le 19 octobre 2022. URL : http:// journals.openedition.org/ideas/425 ; DOI : https://doi.org/10.4000/ideas.425

Ce document a été généré automatiquement le 19 octobre 2022.

\section{(i) $\$$

Creative Commons - Attribution - Pas d'Utilisation Commerciale - Pas de Modification 4.0 International - CC BY-NC-ND 4.0

https://creativecommons.org/licenses/by-nc-nd/4.0/ 


\title{
La politique alimentaire et la consommation dans l'Argentine péroniste
}

\author{
Food Politics and Consumption in Peronist Argentina \\ La política alimentaria y el consumo en la Argentina Peronista
}

\author{
Natalia MILANESIO
}

\section{NOTE DE L'ÉDITEUR}

Cet article est une traduction réalisée, avec l'accord de Duke university Press, par Laura PERTUY de l'article de Natalia MILANESIO, «Food Politics and Consumption in Peronist Argentina ", publié dans The Hispanic American Historical Review, vol. 90, n 1 , February 2010, pp. 75-108.

Je tiens à remercier Konstantin Dierks, Eduardo Elena, Peter Guardino, Jeffrey Pilcher, Ricardo Salvatore et César Seveso pour leurs commentaires utiles sur une version antérieure de l'article. Je salue également le Social Science Research Council et l'American Council of Learned Societies pour avoir soutenu mes recherches et la rédaction de cet article. Traduction de Laura Pertuy. Ont participé à la traduction française : Claire Bouffard, Domingo Garcia-Garza et Cyril Jayet.

1 Une famille de la classe ouvrière se régale d'un dîner copieux. Ravis, les enfants ouvrent grand la bouche pour engloutir de généreuses bouchées. Le pain et le vin, importants symboles d'abondance et d'appartenance communautaire pour ce pays très catholique qu'est l'Argentine, sont disposés à un endroit stratégique de la table. La famille se restaure sous le portrait du président Juan Domingo Perón en tenue militaire de gala. Cette photographie, en couverture du célèbre magazine Ahora, évoque les relations entre la consommation alimentaire, la politique et l'État, tout comme le pouvoir incontestable de la nourriture dans la transmission de sens. L'image que l'on veut donner est celle d'un peuple heureux, d'une race forte et saine, d'une famille unie, 
d'un père capable de subvenir aux besoins des siens, d'une mère nourricière, tout comme d'un gouvernement juste, d'un leader omniprésent, d'une terre riche et d'une économie bien dirigée ${ }^{1}$.

Dès les débuts de l'administration péroniste (1945-1955), la consommation alimentaire représente à la fois un objectif primordial de la politique d'État et un élément central de la propagande officielle. Dans cet article qui examine les aspects structurels et institutionnels caractéristiques de la politique alimentaire péroniste, je me concentrerai principalement sur ce que l'anthropologue Arjun Appadurai a appelé la "virtuosité sémiotique » de l'alimentation, c'est-à-dire sa capacité à transmettre des messages multiples en tant que symbole culturel (Appadurai, 1981: 494). Les chercheurs ont majoritairement étudié les aspects symboliques de l'alimentation et l'intervention des États modernes dans l'approvisionnement alimentaire comme deux sujets séparés. Quant aux universitaires spécialisés dans les thématiques alimentaires, ils ont désigné les classes, ethnies, races, femmes, hommes, agences de publicité, chaînes de restauration, défenseurs de l'alimentation et des consommateurs comme les principaux producteurs et récepteurs de sens via l'alimentation. Les gouvernements sont généralement jugés à l'aune de leur rôle dans la mise en place d'une politique alimentaire efficace ${ }^{2}$. Cet article explore ces rôles, tout comme les usages idéologiques et les implications symboliques de la politique alimentaire de l'État argentin ainsi que ses répercussions sur la consommation.

3 À la fin des années 1940 et dans les années 1950, une augmentation de la consommation de bœuf par personne ébranle l'image de l'Argentine, jusqu'alors considérée comme économie exportatrice soumise au capitalisme étranger. En préférant les consommateurs argentins aux marchés extérieurs, la politique péroniste concernant le bœuf a ainsi développé une puissante idéologie de souveraineté économique. Celle-ci a renforcé l'engagement de l'État à bâtir une nation prospère et industrielle comme à redistribuer la richesse nationale au profit de la population locale. Entre 1946 et 1949, le gouvernement utilise l'augmentation de la consommation en bœuf pour affirmer le nouveau droit de la classe ouvrière à ce qui était auparavant considéré comme de la " nourriture de luxe ». Cette rhétorique était particulièrement significative pour les principaux partisans du péronisme, à savoir les classes populaires urbaines et les migrants de la classe ouvrière nationale, dont la demande en bœuf n'avait jamais été satisfaite par le passé. Le gouvernement péroniste a ainsi tiré profit des notions populaires, scientifiques et historiques qui faisaient du bœuf un élément essentiel d'une alimentation idéale. Il a utilisé la consommation de cette viande pour construire une idéologie plus vaste qui cherchait par différents moyens soutenir le droit des secteurs populaires à revendiquer l'auto-gratification et plus largement, l'amélioration de leur niveau de vie. Aspiration populaire clé, la consommation de bœuf se trouvait au cœur d'une idéologie plus vaste qui visait la hausse du niveau de vie. Elle s'apparentait également à une stratégie plus ambitieuse de la communication d'État sur le droit des secteurs populaires.

Alors que les universitaires spécialisés dans les questions alimentaires ont exploré les changements au sein des habitudes gastronomiques au fil des siècles, l'époque péroniste a été témoin d'une rapide transformation du régime alimentaire populaire et a produit l'exact opposé en moins d'une décennie ${ }^{3}$. Au début des années 1950, une crise économique force le gouvernement à interdire la consommation de bœuf un jour par semaine et à mettre en place une grande campagne afin d'encourager la consommation 
de légumes et de fruits, en même temps qu'elle préconise de substituer le poisson au bœuf. Afin de mettre en œuvre une nouvelle politique alimentaire, le gouvernement s'appuie sur la santé de la population et sur la préservation de la tradition culinaire nationale, les deux principes structurant le nouvel imaginaire alimentaire. Là encore, la politique alimentaire péroniste s'inspire des notions populaires et scientifiques en adoptant l'idée d'une alimentation variée comme base d'un style de vie sain. Ces idées répondent à une inquiétude généralisée par rapport aux changements dans les habitudes alimentaires populaires hors des repas traditionnels. En effet, celles-ci étaient considérées comme les conséquences de l'urbanisation, de la migration interne et de l'industrialisation.

5 La politique, la consommation et les imaginaires alimentaires figurent parmi les aspects les moins développés de l'historiographie argentine. Ils appartiennent aussi à un chapitre inexploré de l'histoire du péronisme. À cause des implications considérables de la politique alimentaire sur le régime péroniste, nous disposons ici d'un cas idéal pour étudier l'invention, l'appropriation et la manipulation des questions alimentaires par l'État. La politique alimentaire était un élément central dans la mise en place de la politique internationale du commerce argentin et de la politique sanitaire publique. De plus, les travailleurs argentins dépensaient plus de la moitié de leur salaire en nourriture tandis que les syndicats réclamaient depuis longtemps une alimentation meilleure et à bas coût. La politique alimentaire péroniste constituait donc un moyen direct de rassembler et de conserver les principaux soutiens du gouvernement, c'est-àdire les classes ouvrières et le mouvement syndicaliste. En fait, l'augmentation de la consommation alimentaire, rendue possible par une augmentation de salaires, le contrôle des prix et l'intervention de l'État dans la production et la distribution des aliments, ont fait de la politique alimentaire un élément clé des stratégies symboliques ou matérielles d'amélioration du niveau de vie des catégories les plus populaires. Le discours sur une vie meilleure basée sur l'alimentation constitue à la fois un élément puissant de la propagande péroniste et un instrument déterminant dans la construction de sa légitimité politique.

6 Cet article s'inscrit dans un long travail de réflexion sur l'étude de ce que le sociologue Juan Carlos Torre et l'historienne Elisa Pastoriza ont appelé «la démocratisation du bien-être » dans la période péroniste. Les universitaires spécialistes du péronisme ont commencé à examiner ce processus en s'intéressant aux politiques du logement, du tourisme, de la sécurité sociale et de la consommation. D'autres ont fait de même en analysant les projets de représentation et d'iconographie du régime autour de quelques-unes de ces questions (Torre et Pastoriza, 1998 : 257-312 ; Aboy, 2005 ; Ballent, 2005 ; 2005 ; Plotkin, 2003 ; Scarzanella, 1998 : 65-84 ; Elena, 2002). Dans cet article, je vais à l'encontre des analyses qui ont tendance à séparer le politique et de l'imaginaire afin d'ouvrir de nouvelles pistes de réflexion sur le péronisme. Tout d'abord, je montre le rôle central des dimensions économiques, politiques et iconographiques de l'alimentation dans la définition de la politique publique, de la culture commerciale, de la santé publique et du bien-être social, national et physique. Grâce à ce nouvel intérêt porté à l'alimentation, je suis en mesure de réinterpréter le nationalisme et le programme social, des concepts clés de l'idéologie péroniste pour les chercheurs. Enfin, mon analyse démontre l'importance du rôle joué par l'État péroniste dans la résolution des problèmes nutritionnels et l'éducation diététique de la population. L'État s'intéressait à l'appropriation et à la redéfinition d'arguments sur l'alimentation 
nationale déjà présents dans la culture populaire ainsi que dans le discours médical de "gauche».

\section{Consommation de bœuf et nationalisme}

7 En 1949, le Ministère de la Santé organise une conférence attendue sur la politique alimentaire argentine, en présence d'importants spécialistes des secteurs de la nutrition, de l'agronomie, de l'hygiène publique ainsi que des dirigeants de toutes les agences gouvernementales liées à la production, la distribution et la consommation alimentaires. Dans son discours inaugural, Perón affirme que « plus personne ne meurt de faim... Des salaires justes permettent désormais à l'ouvrier de mieux aménager sa cuisine et de récupérer complètement de sa fatigue. Un ouvrier et sa famille consomment actuellement presque deux fois la quantité de nourriture qu'ils consommaient il y a six ans $»^{4}$. Perón énonce ainsi les deux façons dont son gouvernement envisage la consommation alimentaire. Premièrement, comme un élément servant à mesurer la façon dont les ouvriers en Argentine péroniste perçoivent des salaires honnêtes et disposent d'heures de travail adéquates. Dans un deuxième temps, le gouvernement conçoit la consommation alimentaire comme un indice de la satisfaction du peuple par rapport aux privations du passé. Cette même consommation est ainsi devenue un élément clé de la justice sociale préconisée par le programme péroniste et la matérialisation du droit de l'ouvrier au bien-être garanti par la constitution péroniste de $1949^{5}$.

Un accès aisé à une alimentation savoureuse, bon marché et disponible en abondance leur semblait fondamental pour plusieurs raisons. Premièrement, les familles de la classe ouvrière issues des sociétés industrielles consacraient plus de la moitié de leurs salaires à la consommation alimentaire, même si cette proportion diminuait à mesure que les salaires augmentaient. Deuxièmement, l'alimentation à bas coût et en quantité suffisante constituait une demande historique, bien que non satisfaite, de la classe ouvrière argentine. En 1942, le leader communiste Paulino González Alberdi déclare ainsi: «le manque de nourriture est un malheur qui s'est abattu sur les foyers argentins. C'est la douleur d'une mère qui se voit forcée de marchander pour le pain et le lait nécessaires à ses enfants car elle n'a pas assez d'argent». La demande de nourriture à petit prix et en quantité suffisante se présente comme la contrepartie aux revendications urgentes pour de meilleurs salaires et aux avertissements sur la malnutrition répandue des ouvriers, que par ailleurs les élites ont systématiquement ignorés (Morse Woodbury, 1942: 5 ; González Alberdi, 1942:5) ${ }^{6}$. De manière tout aussi importante, l'accès du peuple à des quantités adéquates de nourriture bon marché était vital à l'accomplissement du programme péroniste d'industrialisation nationale. L'alimentation constituait un élément central pour d'autres projets populistes latinoaméricains, par exemple au Mexique sous l'administration de Lázaro Cárdenas (1934-1940). Tant en Argentine qu'au Mexique, l'intervention de l'État pour assurer l'approvisionnement adéquat en nourriture et des prix décents était essentielle afin de maintenir des salaires peu élevés dans les usines et permettre la production de biens de consommation aux prix abordables. De plus, la politique alimentaire encourage indirectement la consommation de biens manufacturés. En effet, en garantissant des aliments à bas prix, l'État réduit la forte proportion du budget consacrée à l'alimentation par la classe ouvrière (Ochoa, 2000 ; Wright, 1985 : 24-45). 
9 Le contrôle des prix a ainsi permis l'augmentation de la consommation alimentaire annuelle tandis que les salaires, tandis que la hausse de salaires a eu un impact direct sur le marché. De 1945 à 1949, les salaires augmentent en termes réels de 62\%. En 1949, le volume des ventes des épiceries est presque $40 \%$ plus élevé qu'au début de la décennie. L'augmentation des salaires, parallèlement à d'autres facteurs, comme l'augmentation des dépenses gouvernementales, finit par aggraver l'inflation préexistante (Gerchunoff et Llach, 1998: 181)7. Pour combattre cette inflation, de nombreux décrets et lois fixent des prix maximums, des niveaux de profit limités pour les industriels, ordonnent des inspections de magasin, donnent des amendes et incarcèrent les propriétaires de magasins qui ne se plient pas à ces régulations. De plus, le gouvernement ouvre des marchés municipaux qui vendent de la nourriture à prix réduits et soutiennent les associations de consommateurs. Au même moment, la Fondation Eva Perón lance ses proveedurías, des magasins qui offrent des biens à faibles prix (Elena, $2007: 111-49$ ).

10 Le gouvernement déclare fièrement que le changement principal dans les habitudes alimentaires populaires tient au fait que les Argentins doublent presque la consommation annuelle de bœuf recommandée. Ramón Carrillo, le Ministre de la santé, affirme qu'un citoyen argentin consomme 150 kilos de bœuf par an, alors que la quantité idéale se situe à 87 kilos. Selon les chiffres de Carrillo, les Américains ne consomment pas plus de 70 kilos par personne par an, tandis que les Italiens et les Espagnols n'en consomment que 17. Ces quantités font de l'Argentine le plus gros consommateur de bœuf au monde ${ }^{8}$. Ce constat est accueilli comme une bonne nouvelle. En effet, depuis les années 1930, les discours scientifiques sur l'alimentation conforte l'idée selon laquelle le bœuf constitue la base idéale d'une alimentation riche en protéines (Escudero, $1934: 79$ ).

11 L'État péroniste soutient les usines de conditionnement de viande via des subventions aux ventes de bœuf dans les villes principales, impose un plafond des prix, ferme les abattoirs illégaux et combat les marchés clandestins. En 1950, le Congrès vote une loi pour contrôler et coordonner toutes les questions liées à la production et à la distribution du bœuf ; cette même loi mène à la création de l'Institut de l'élevage de bétail argentin (rattaché au Ministère du Commerce), suivi par la création de l'Institut National de la viande un an plus tard. Dans le but de servir le marché national de façon plus efficace, la loi met en place des subventions gouvernementales à cette filière, dont l'Association des producteurs de viande argentins, le Marché de Liniers (le marché de bétail le plus important du pays) et l'abattoir municipal de Buenos Aires (Peffer, 1960 : 151-84, $1961: 33-75)$.

12 Même avec des salaires corrects et des prix accessibles, l'augmentation de la consommation annuelle en bœuf par personne aurait été impossible sans la disponibilité de cette même viande. Depuis les années 1920, socialistes et communistes liaient les pénuries et la valeur excessive du bœuf sur le marché national à l'emprise des capitaux britanniques et américains sur l'industrie de la viande. La filière bovine d'Argentine veillait à assurer des quotas d'exportation en bœuf importants, à faibles prix et de grande qualité, au détriment du consommateur local. Dépendante des marchés internationaux et financée depuis l'étranger, cette industrie représentait l'asservissement de l'oligarchie argentine aux intérêts étrangers et au pouvoir du lobby britannique. On peut s'apercevoir de ce phénomène de manière flagrante dans l'accord, grandement méprisé, de « Roca-Runciman » signé en 1933 avec la Grande-Bretagne, qui 
limitait à seulement $15 \%$ la part d'exportation pouvant être gérée par des abattoirs nationaux. L'accord garantissait aussi que les livres sterling récoltées grâce au commerce du bœuf devraient être dépensées en Grande-Bretagne, afin d'importer du charbon, du matériel ferroviaire, des textiles, pour lesquels un tarif préférentiel avait été établi ; il stipulait également le versement des profits aux entreprises britanniques (de la Torre, 1947). Selon les communistes, alors que l'Argentine était le « ravitailleur de la Grande-Bretagne ", le bœuf représentait un aliment de luxe pour les ouvriers locaux. Du milieu des années 1910 au milieu des années 1920, 90\% des exportations de bœuf frais étaient destinées à la Grande-Bretagne. Ce chiffre venait appuyer l'opinion de certains observateurs selon laquelle l'Argentine était devenue « l'empire informel de la Grande-Bretagne » (Smith, $1969: 1$, 35 ; Ferns, $1953: 60-75)^{9}$.

13 Cela dit, le gouvernement péroniste a fait du commerce de bœuf un exemple de la transformation de l'Argentine en une nation "socialement juste ", "politiquement souveraine " et " économiquement indépendante ", autrement dit les leitmotivs traditionnels du péronisme. En s'appuyant sur la question du bœuf, l'administration s'est fait le garant idéal du pouvoir alimentaire en Argentine. L'Accord Eady-Bramuglia de 1946 est le premier traité conclu entre la Grande-Bretagne et le gouvernement péroniste. Les parties concernées décident que même si la Grande-Bretagne a formulé une demande pour récupérer la totalité du surplus de bœuf, l'Argentine doit conserver $17 \%$ des exportations de bœuf pour pouvoir négocier avec les autres acheteurs dans la première année du traité et $22 \%$ des exportations dans la deuxième année. La GrandeBretagne achèterait ces quotas uniquement dans le cas où l'Argentine ne parviendrait pas à les vendre. Le traité établit aussi une augmentation de prix de $45 \%$ par rapport à celui fixé dans le traité précédent en 1939. En 1948, les gouvernements argentins et britanniques signent l'Accord des Andes dans lequel l'Argentine consent à vendre à la Grande-Bretagne 420000 tonnes de bœuf par an. Moins d'un an plus tard, les gros titres britanniques accusent le gouvernement péroniste d'opportunisme vénal, alors que les journaux argentins soutiennent le Président dans sa défense de l'intérêt national. La presse locale proclame, enthousiaste, que l'Argentine n'est en aucun cas une colonie britannique et même qu'elle a atteint «l'âge adulte politique». À l'origine de ces opinions adverses, la demande formulée par le gouvernement argentin d'instaurer des prix plus élevés pour les 75000 tonnes du quota original. Il s'appuie sur le fait que les prix établis en 1948 sont extrêmement bas. En fait, le gouvernement a subventionné des éleveurs et des usines de conditionnement de la viande afin de pouvoir fournir les 345 000 tonnes promises aux Britanniques. Perón prétend que les conditions désavantageuses de l'accord forcent l'Argentine à vendre une partie du quota britannique à d'autres marchés européens pour s'assurer un certain niveau de profit et obtenir des importations nécessaires que la Grande-Bretagne est incapable de fournir. Qui plus est, la consommation nationale de bœuf a atteint des niveaux jamais vus jusque-là et les consommateurs argentins paient désormais des prix plus élevés que ceux que la Grande-Bretagne a accepté de payer dans l'Accord des Andes (Licéaga, $1952: 231-32,269-71) .^{10}$

14 Le conflit avec la Grande-Bretagne au sujet du quota d'exportation du bœuf fait suite à un autre épisode très médiatisé du nationalisme péroniste lié aux intérêts britanniques. En 1947, l'Argentine acquiert les compagnies ferroviaires britanniques grâce à ses livres sterling «gelées" à Londres pendant la guerre. L'opposition critique cette nationalisation car elle décapitalise les entreprises, les rend obsolètes et de moins en moins profitables. Cependant, l'Argentine profite pleinement de cette situation puisque 
les livres ne sont pas convertibles en dollars. De plus, la crise financière que traverse la Grande-Bretagne et la taille de sa dette rendent impossible un éventuel remboursement ${ }^{11}$. La négociation, fêtée avec emphase comme une partie du programme péroniste d'indépendance économique, représente la fin d'une époque dans laquelle les Britanniques utilisent leurs trains pour financer l'acheminement à bas frais du bœuf vers les ports en imposant des taxes sur l'expédition des aliments et biens industriels destinés au marché intérieur (Perón, 1984 : 103).

L'administration péroniste ne contestait pas le rôle de l'Argentine comme fournisseur pour le marché alimentaire international. Cependant, le gouvernement subordonnait ce rôle à l'industrialisation nationale, comme le montre le premier programme quinquennal, qui garantissait le soutien financier et la protection légale à l'industrie, et l'Institut de promotion du commerce argentin (IAPI). L'IAPI destinait la différence des prix payés aux producteurs ruraux et les sommes obtenues à partir des ventes internationales, au secteur industriel. Jusqu'en 1949, l'IAPI arbitre les ventes de bœuf frais et de bœuf en conserve entre les producteurs locaux et les marchés étrangers. Cette année-là, à cause des désagréments suscités par le Traité des Andes et la dévaluation de la livre sterling, l'IAPI réduit sa participation au commerce du bœuf frais et laisse l'exportation du bœuf en conserve au secteur privé. L'IAPI n'est qu'un intermédiaire qui négocie les prix et quotas, tandis que l'industrie s'adresse directement aux acheteurs internationaux. Après 1949, l'IAPI devient la source la plus importante de soutien financier pour les éleveurs et l'industrie de conditionnement de la viande (Novick, 1986 : 57-60).

16 En donnant la priorité aux consommateurs nationaux plutôt qu'au marché britannique, le gouvernement péroniste remet en question la dichotomie traditionnelle « pays riche - population pauvre » qui dominait l'imaginaire populaire ; il synthétise également les discours portant sur la spoliation nationale par des pays étrangers (Horowitz, 2006 : 18). En un sens, le bœuf représente symboliquement l'aspiration de la classe ouvrière à une vie meilleure aux États-Unis et en Grande-Bretagne, tout autant qu'en Argentine. Cependant, en Argentine, les intellectuels nationalistes et la gauche considèrent que cette aspiration demeure insatisfaite à cause de l'asservissement des élites nationales aux capitaux étrangers. Cette position a placé le pays dans une situation plutôt désavantagée au sein de l'économie mondiale. Le péronisme conteste l'image qui rapprochait le bœuf d'un courant de domination étrangère et de nécessité inatteignable ; au contraire, il le considère nécessaire au confort national et à l'indépendance économique. Le magazine Caras y Caretas illustre parfaitement ce processus. Sous le titre « Ils s'immiscent depuis l'étranger pour dévorer notre nourriture », on aperçoit un John Bull en surpoids, pareil à un ogre et véritable personnification de l'Anglais type, s'attaquer à un énorme steak avec grand appétit. Sous sa table, un paisano maigrichon et rapetissé regarde Bull sans espoir, les bras croisés. Un os se trouve dans une assiette posée sur sa petite table. Bull commente : «Mon ami, tu devrais être fier de ton pays et du bœuf aussi délicieux que bon marché qu'il produit ». Le paisano répond : « Pour moi, ces avantages sont peau de chagrin ». Sur la page suivante, sous le titre «Les étrangers auront seulement nos restes ", Juan Pueblo, un ouvrier musclé et en bonne santé, porte le badge péroniste et savoure un steak juteux. Sous sa table, un John Bull squelettique, pareil à un cadavre, est assis devant une assiette vide. L'ouvrier, dont la ressemblance avec Perón est surprenante, commente : «Mon ami, nous sommes fiers de notre pays ; 
il produit du bœuf délicieux et bon marché ». Bull lui répond : «Juan Pueblo, mon ami, lorsque tu auras fini de manger, me vendras-tu tes restes ? $»^{12}$.

Ces deux images opposent la privation de la société agraire pauvre, qui nourrit le marché international à ses propres frais, à un pays industriel riche qui privilégie le bien-être de sa population aux dépens de ses intérêts à l'étranger. Les statistiques montrent la manière dont la distribution de bœuf est destinée au marché extérieur au

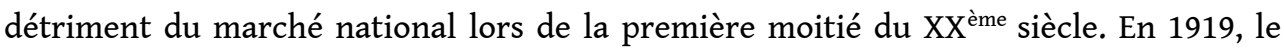
marché intérieur reçoit $42,7 \%$ de la production de bœuf du pays (431 155 tonnes) alors que 57,3\% est exportée (579 716 tonnes). À l'inverse, en 1949, les Argentins consomment $76,7 \%$ de la production totale (1536126 tonnes) et exporte seulement 23,3\% (467 076 tonnes) (Gerchunoff et Llach, $1998: 192-95)^{13}$.

La politique péroniste par rapport au bœuf et sa puissante rhétorique nationaliste transmettaient un message masculinisant aux Argentins. En Argentine, comme aux États-Unis et en Grande-Bretagne, le lien entre bœuf et masculinité est profondément enraciné dans l'histoire. En effet, l'asado (barbecue) traditionnel, des morceaux de bœuf cuits sur une parrilla (un grill) ou directement sur la braise, est le plat national le plus ancien, principalement de la Pampa, et dont on peut retracer les origines à la période précédent l'indépendance. C'est le plat fétiche du gaucho, figure de la masculinité argentine à la fois libre et courageuse ${ }^{14}$. Dans l'imaginaire populaire, le tempérament du gaucho est intrinsèquement lié à une alimentation entièrement basée sur la consommation de bœuf. Depuis le XIX ${ }^{\text {ème }}$ siècle, les autochtones, aussi bien que les étrangers, attribuent la force physique des gauchos au bœuf, avançant que cet aliment « est le secret de leur vigueur traditionnel, ce qui les rend forts et courageux $»^{15}$. Cette croyance, appuyée par le discours médical, stimule la prédilection des hommes pour le bœuf. De plus, l'asado est selon la tradition le seul plat préparé par l'homme. Cette activité qui requiert la manipulation de viande crue, de couteaux et de feu, est donc associée à une véritable compétence masculine. En garantissant l'approvisionnement de bœuf pour le marché national, l'administration péroniste renoue symboliquement avec la dimension masculine de l'alimentation traditionnelle argentine ${ }^{16}$.

19 À la fin des années 1930, l'intellectuel nationaliste Raúl Scalabrini Ortiz s'interroge sur les intérêts de la Grande Bretagne en Argentine et à la politique péroniste concernant la filière de viande de bœuf en les considérant comme " la tragédie nutritionnelle de notre pays ». Il avance l'idée selon laquelle « le peuple argentin mange peu et vit dans la pauvreté pour que les Britanniques puissent manger copieusement et vivre bien » (Scalabrini Ortiz, 1940 : 196-97). Dix ans plus tard, le gouvernement péroniste remet en cause le lien entre la commercialisation alimentaire pour l'exportation et la rareté locale, et donc les rapports hiérarchiques entre les pays qui produisent la nourriture et ceux qui la consomment. Le péronisme réarticulait la relation entre la conception du bien-être populaire et le marché britannique grâce à la politique bovine du péronisme. Celle-ci transmettait des messages nationalistes défendant l'auto-détermination économique, d'industrialisation, de souveraineté, de masculinité, tout en glorifiant les nouvelles quantités de bœuf consommées par les Argentins.

\section{Luxe et Droit}

20 La redistribution entre les marchés locaux et internationaux a modifié la consommation de bœuf par personne en Argentine. En 1948, la consommation annuelle 
par personne à Buenos Aires atteint 103,2 kilos. Bien que ce soit 50 kilos de moins que la quantité évoquée par Carrillo, les statistiques officielles s'intéressent toujours à la croissance de la consommation de bœuf par rapport à la consommation par personne en 1935, laquelle s'élève alors à 61,2 kilos $^{17}$. Malheureusement, au-delà des revendications de l'administration par rapport au changement sans précédent qui affectait l'alimentation de la classe ouvrière, les statistiques des années péronistes sont rares. Relativement incomplètes, elles ne font pas la distinction entre la consommation de bœuf dans les différentes classes sociales. Cependant, un rapport de la FAO (Food and Agriculture Organization of the United Nations) daté de 1950 avance que la consommation de bœuf a grimpé sous le gouvernement péroniste et que, pendant cette période, la proportion de bœuf consommée par les Argentins était l'une des plus élevées du monde (Gustavino, $1954: 14)^{18}$.

21 Le gouvernement utilise l'excès de consommation de bœuf dans l'alimentation nationale comme argument pour promouvoir l'accès des classes populaires à ce qui était auparavant de la «nourriture de luxe ». Perón déclare : « Nous avons déjà corrigé bien des défauts... grâce à notre politique sociale. En augmentant le niveau de vie de la classe ouvrière, nous avons permis aux gens de manger plus et d'acheter certains types d'aliments qui étaient auparavant considérés comme des articles de luxe réservés aux riches $»^{19}$. La définition du bœuf comme aliment de luxe remontait à l'arrivée des immigrés d'Europe et à la transformation de leur alimentation sans viande - dépourvue de protéines, principalement végétarienne et globalement pauvre - par la consommation de bœuf ${ }^{20}$. L'équation entre bœuf et luxe renvoyait aussi au langage que communistes et socialistes avaient utilisé pour faire entendre leurs réclamations à l'égard de cette denrée et dénoncer les prix exorbitants pratiqués sur les 25 années précédentes. Quelques années avant l'élection de Perón, le leader communiste González Alberdi avait affirmé que «les tarifs élevés du bœuf constituent l'un des aspects du niveau de vie élevé dont souffre la classe ouvrière. Traditionnellement, et grâce à sa valeur nutritionnelle, le bœuf a joué un rôle important dans le régime alimentaire des Argentins. Cependant, le prix élevé du bœuf a fait du steak - et même de l'os à moelle utilisé pour les ragouts - des aliments de luxe $»^{21}$. Enfin, en cette période d'aprèsguerre, la définition du bœuf comme aliment de luxe associe des conceptions de richesse nationale à des discours qui opposent les secteurs populaires bien-nourris de l'Argentine péroniste aux masses affamées des pays d'Europe ravagés par la guerre ${ }^{22}$.

En réalité, la consommation de bœuf reste négligeable sous le péronisme, notamment dans les régions du nord et du sud de l'Argentine, où l'on mangeait plus de maïs et d'agneau que dans le reste du pays. Dans ces zones, la demande en bœuf est moindre à cause des prix élevés, de la rareté de cette viande et d'une distribution inefficace. Alors que l'administration péroniste se félicite d'une augmentation moyenne dans la consommation alimentaire et d'une alimentation plus calorique dans les régions centrales, les populations des provinces du nord et du sud souffrent encore de malnutrition. De plus, la famine représente une véritable souffrance que le gouvernement ignore la plupart du temps (Pierángeli, 1951 : 91-103) ${ }^{23}$. Par contre, dans le Cuba des années 1960, la révolution échoue à augmenter la consommation alimentaire moyenne, la consommation calorique par personne décline, et de fait, plus de personnes se retrouvent à consommer moins. Cependant, un système national de distribution et de rationnement efficace garantit assez de nourriture pour éradiquer virtuellement la malnutrition de l'île (Foster et Handelman, 1985 : 174-98). 

en mesure de produire leur propre nourriture. Si l'on compare aux citadins et aux personnes vivant dans d'autres régions du pays, la population rurale des pampas avait généralement un accès plus aisé à une variété de produits basiques bon marché, dont le bœuf. Même si, depuis les années 1920, les médecins alertaient les élites politiques de la sous-alimentation endémique de la population, particulièrement dans le nord, la consommation argentine de bœuf demeurait remarquablement plus élevée qu'aux États-Unis et dans d'autres pays d'Amérique latine (Schleh, 1930 ; Llorens, 1942: 74, 1944). En fait, la tradition coloniale de l'asado montre la place centrale du bœuf dans le régime alimentaire du peuple argentin. Dans l'imaginaire collectif, la famine était une notion inconnue, car le gaucho pampeano du début du XIX ème siècle incarnait une période d'accès gratuit et en grandes quantités aux animaux sauvages, parmi lesquels le bœuf. On retrouve alors l'idéalisation d'une époque qui avait précédé la consolidation de grandes propriétés, la mise en place d'usines de conditionnement de la viande et l'émergence d'un réseau de distribution alimentaire; autant de changements qui ont rendu difficile l'achat de bœuf étant donné son coût élevée ${ }^{24}$. du sens pour un public d'ouvriers, de migrants du pays et de citadins pour qui il était devenu une denrée de plus en plus inatteignable. Cet argument était particulièrement significatif pour les citadins qui représentaient près de $62 \%$ des habitants d'Argentine (Girbal-Blacha, 2001: 87). De façon plus précise, avec autour de 30\% de la population nationale, le Gran Buenos Aires (la capitale et les municipalités alentour) consommait plus de $36 \%$ de la production totale de bœuf ${ }^{25}$. Au sein de cette population, l'idée selon laquelle la consommation de bœuf était un luxe parlait particulièrement à la classe ouvrière. Tout au long du début $\mathrm{du} X \mathrm{XX}^{\text {ème }}$ siècle, des ouvriers porteños déconcertés ont été témoins de la réduction des portions de bœuf destinées au marché intérieur à cause de la hausse de prix, de la diminution de qualité et de la réduction des stocks. Tous ces facteurs étaient principalement des conséquences de la participation croissante de l'Argentine au commerce international du bœuf (Ferreras, 2001 : 157-80, 173). En 1947, 73\% d'ouvriers porteños étaient des migrants venus d'autres provinces. Pour les migrants du nord et du sud, provenant des régions défavorisées, le bœuf représentait donc un luxe. En effet, ils avaient souffert de la "famine de bœuf» de manière systématique (Germani, $1973: 432-88,452)^{26}$. Les statistiques officielles montrent que, arrivés à la fin des années 1930, la femme et les trois enfants d'un ouvrier non qualifié vivant à Buenos Aires, consommaient 37 kilos de bœuf par mois. À La Rioja, à San Luis, et à Santiago del Estero, une famille de la même taille en consommait seulement 20 kilos par mois. ${ }^{27}$ Certains observateurs se montraient moins optimistes. Pedro Escudero, pionnier de la recherche alimentaire en Argentine, rappelle l'expérience déchirante de sa visite à San Juan au début des années 1930. Il y a découvert que l'alimentation quotidienne des classes populaires était composée de raisin, l'aliment de base de la région, et d'un ragoût de viande de chien ${ }^{28}$.

L'association entre bœuf et luxe soulignait l'intérêt officiel pour le péronisme qui avait inauguré une période où la hausse du niveau de vie prévalait sur la protection des besoins humains basiques. Le cidre et le pan dulce, l'alcool et le dessert traditionnels des fêtes de Noël et du Nouvel An argentins, sont devenus des icônes de ce processus. Alors que l'opposition déclamait, « Nous ne considérons pas le pan dulce ou le cidre comme des besoins humains basiques ", le gouvernement péroniste proclamait le droit aux 
mets de Noël pour tous, même les plus pauvres, et l'inscrivait au rang de compensation historique pour les privations passées ${ }^{29}$. Au moins jusqu'au début des années 1950, lorsque les problèmes d'inflation et d'équilibre commercial entrainent une relative période d'austérité économique, la nouvelle prérogative populaire aux signes de richesse englobe ne seulement l'alimentation mais aussi les vêtements, les loisirs et le tourisme (Pastoriza, 2002) ${ }^{30}$. Les programmes d'assistance sociale dirigés par la Fondation Eva Perón mettent en exergue le nouvel accès du peuple à ce qui était considéré comme un luxe. En 1955, un rapport rédigé par la Revolución Libertadora, qui a par ailleurs renversé Perón, indique que la nourriture et les vêtements fournis aux enfants nécessiteux par la Fondation sont un exemple de "somptuosité " (cité dans Duarte, 1972 : 92). Le Hogar de la Empleada General San Martín, situé dans le centre-ville de Buenos Aires, en est l'un des exemples les plus frappants ; il offre également un logement à bas coût aux ouvrières. Ouvert par la fondation en 1949, le Hogar est un immeuble richement décoré qui comprend un restaurant splendide et abordable, en libre accès ${ }^{31}$. Les cantines de l'État au Pérou et au Mexique se caractérisent par une architecture sobre, une décoration basique, des tables simples, des lavabos communs et spartiates ainsi qu'un service qui s'apparente à celui d'une cafétéria. À l'inverse, le restaurant péroniste, avec ses maîtres d'hôtel et ses serveurs bien élevés, ses menus riches et variés, sa décoration stylée et ses tables élégantes, reflète les efforts déployés par l'État pour offrir au public un dîner plaisant et raffiné (Drinot, 2005 : 245-70 ; Aguilar-Rodríguez, 2007 : 177-205).

Grâce à la mise en avant de la consommation alimentaire, le gouvernement péroniste a fait de l'hédonisme une aspiration légitime des secteurs populaires. Une brochure de l'une des maisons de la «Fondation pour femmes célibataires » informait ses lectrices que «le seul avantage de la nourriture n'est pas d'être abondante et nutritive. La présentation des plats est également une vraie préoccupation car, malgré le désir de minimiser l'importance de manger, cette action va bien au-delà de la satisfaction du besoin miséreux d'apaiser la faim. En effet, les sens contribuent au plaisir gustatif, et une jolie salle à manger embellie par des fleurs et de la lumière, en plus d'une succession de plats variés et bien présentés, contribuent à notre plaisir tout en nous ouvrant l'appétit et en aidant à la digestion des aliments consommés ${ }^{32}$. Le discours péroniste a ainsi fait passer l'action de manger d'une simple pratique de subsistance à une expérience sensorielle agréable. Autrefois considérée comme le carburant du corps, la nourriture est devenue une source de plaisir. Cette prérogative, autrefois monopolisée par les groupes sociaux les plus aisés, incarnait la contrepartie aux descriptions officielles de l'oligarchie vénale "qui n'avait connu que les luxes d'une table recouverte de mets, les chuchotements de la soie et les voyages de plaisance à l'étranger » tout en condamnant les travailleurs à la privation et à la souffrance (cité dans Bianchi et Sanchís, $1988: 59)^{33}$.

L'alimentation transmettait, grâce à l'accès différentiel aux aliments, des messages d'indulgence, d'accès au bonheur et de réduction des inégalités sociales. Bien que des niveaux élevés de consommation en bœuf demeuraient largement régionalisés et que la malnutrition continuait à affecter des zones considérables du pays, la rhétorique péroniste concernant les aliments de luxe abordables était prépondérante. Elle capitalisait sur une tradition préexistante qui identifiait certains aliments à la richesse et à la prospérité, et sur une famine historique, à la fois littérale et symbolique, avec des aliments peu disponibles aux prix élevés. Parallèlement au discours officiel soulignant la fin d'une époque de privilège social, la notion qui faisait du bœuf un 
aliment luxueux célébrait la popularisation de ce qui avait autrefois été exclusif tout comme la démocratisation d'un bien jadis coûteux. De fait, la consommation de bœuf a contribué au développement d'un sens du droit croissant, également présent dans les discours sur les acquis sociaux récents comme l'accès au logement, aux vacances et aux loisirs. Au vu du taux élevé de la consommation de bœuf par personne chez certains consommateurs, cette transformation ne résulte pas d'une redistribution de nourriture au détriment des secteurs privilégiés ou d'une socialisation de la richesse privée. Elle ne sous-entend pas non plus un nivelage social par l'homogénéisation d'habitudes alimentaires de différents secteurs. De plus, dans sa transition d'un bien de luxe à un bien accessible à tous, le bœuf est devenu le symbole d'un niveau de vie élevé, qu'un moyen de favoriser la mobilité sociale.

\section{Santé, consommation de poisson et mutation de l'imaginaire alimentaire}

Socialistes et communistes ont contesté l'interprétation du gouvernement en avançant que les fruits, les légumes et les œufs constituaient les vrais luxes du budget de la classe ouvrière : ils étaient extrêmement chers et disponibles en petites quantités. Les antipéronistes préféraient une définition du luxe basée sur les prix élevés ainsi que sur l'indisponibilité et déclaraient que ces facteurs, plus que la préférence des consommateurs, forçaient les gens à consommer de grandes quantités de bœuf. Si l'on en croit ce point de vue, le bœuf n'était pas un luxe, mais la seule option dont disposaient les classes populaires ${ }^{34}$. Certains chiffres aident à expliquer en quoi les fruits frais étaient considérés comme des «biens somptueux ». En 1933, une famille de la classe ouvrière avec trois enfants dépensa $\$ 13.44 \mathrm{~m} / \mathrm{n}$ par mois pour 24 kilos de bœuf et $\$ 1.62 \mathrm{~m} / \mathrm{n}$ pour six douzaines et demi de fruits. En 1949, la même famille dépense presque deux fois ce montant pour l'achat de bœuf et presque 10 fois plus pour la même quantité de fruits ${ }^{35}$.

Lors de la conférence de 1949 sur la politique alimentaire, le gouvernement reconnait que le régime alimentaire populaire manque "d'aliments protecteurs", concept générique désignant les sources principales de minéraux et de vitamines comme le lait, les oeufs, les légumes frais et les fruits. Le Ministre Carrillo révèle que les Argentins consomment 50 litres de lait, 190 kilos de légumes et 22 kilos de fruits de moins que la consommation idéale annuelle recommandée. La rareté des aliments protecteurs sur la table de la classe ouvrière s'apparente à la continuation d'une alimentation populaire inadéquate que les chercheurs dénoncent depuis les années 1920. L'administration désigne la production insuffisante, la distribution inefficace, les intermédiaires commerciaux vénaux et le manque d'information publique sur la nutrition comme les raisons principales de ce déficit. Les spécialistes suggèrent différentes manières d'améliorer la production et la consommation d'aliments protecteurs : des contrôles de prix plus stricts, une maîtrise de la distribution, une reconnaissance officielle pour les fermes et les coopératives, de l'aide financière pour l'incorporation d'innovations technologiques dans les petits vergers et les potagers ainsi qu'une éducation alimentaire pour le peuple ${ }^{36}$.

30 Lors de la conférence de 1949, le gouvernement présente un diagnostic sur les habitudes alimentaires en Argentine en se basant sur deux arguments : les Argentins consomment du bœuf de façon excessive, et par conséquent leur consommation 
d'aliments protecteurs est insuffisante. Même si la redistribution des salaires a assuré des repas abondants, le problème principal tient au fait que les familles de la classe ouvrière ne mangent pas nécessairement mieux. Comme seul résultat: un régime déséquilibré nocif pour le corps, la famille et la nation. Le gouvernement établit une liste des conséquences nuisibles de ces pratiques alimentaires : l'obésité, les maladies de l'estomac, des reins et du foie, les problèmes de croissance, le diabète, et des problèmes de pression artérielle. Ces effets demeurent une telle priorité pour le gouvernement que sur les trois années suivantes, et puis à nouveau en 1955, il organise de grandes conférences très médiatisées sur la politique alimentaire nationale ${ }^{37}$.

Bien que les deux aspects de ce diagnostic soient étroitement liés, le gouvernement pointe progressivement le surplus de consommation de bœuf, 60 kilos au-dessus de la quantité annuelle recommandée par personne, comme l'origine des problèmes de santé dont souffre la population argentine. Pour résoudre ce conflit et réduire la consommation de bœuf, le gouvernement lance une campagne officielle intensive, « La Cocina de la Salud» (Cuisine saine). L'administration péroniste bombarde le public d'émissions, de milliers d'articles et publicités dans les magazines et journaux, de posters, de cours de cuisine, de brochures sur la nutrition et d'expositions culinaires. De plus, le Ministère de la Santé met en place une hotline qui, en 1952, reçoit 1455385 appels de demande d'informations sur la recette recommandée du jour. Depuis le début $\mathrm{du} \mathrm{XX}{ }^{\text {ème }}$ siècle, les experts s'accordaient à dire que la population, et particulièrement les secteurs populaires, ignorait la valeur nutritionnelle de la nourriture, avait des pratiques culinaires proches du gaspillage qui resserraient les budgets déjà modestes, et adoptait des habitudes alimentaires malsaines qui affaiblissaient les générations futures. De fait, l'éducation nutritionnelle et les conseils culinaires offerts par l'Etat péroniste ont reçu l'approbation chaleureuse de la communauté médicale qui demandait ces mesures depuis des décennies (Escudero, $1934: 268$; Schleh, 1930) ${ }^{38}$.

Comme le gouvernement avait associé l'alimentation à un niveau de vie plus élevé, et plus particulièrement à des proportions de bœuf plus importantes, la campagne sanitaire visant à réduire la consommation de bœuf était considérée comme une stratégie inattendue et impopulaire. Pour offrir une explication à ce revirement, l'opposition ne tarde pas à souligner la « coïncidence suspecte » entre l'inquiétude du gouvernement envers la santé de la population, couplée à l'excès de bœuf dans l'alimentation populaire, et l'état inquiétant de l'équilibre commercial du pays qui nécessitait l'augmentation des exportations de viande. De 1949 à 1950, et ensuite de 1951 à 1952, la campagne argentine souffre d'une sécheresse qui entraine de terribles conséquences pour l'industrie du bétail ${ }^{39}$. Le bétail meurt de soif et les pâtures dépérissent ; les ranchmen connaissent alors des difficultés à nourrir les troupeaux et les périodes d'engraissement se rallongent. Afin de répondre à la demande nationale croissante, les producteurs abattent veaux et génisses, affectant ainsi gravement le cycle de reproduction. Peu encline à augmenter les prix officiels du bœuf pour abaisser la demande, l'administration endosse la responsabilité des déficits de l'industrie du bétail et subventionne les ventes de bœuf. Le bétail est toutefois décimé d'une manière aussi grave que rapide. À partir de 1950, les consommateurs argentins souffrent de pénuries de bœuf et paient des prix plus élevés que les prix officiels, tandis que le gouvernement rencontre des difficultés à contrôler le commerce local. En effet, la demande insatisfaite entraine l'abattage clandestin et le fleurissement d'un marché de viande clandestin (Peffer, $1960: 160,1961: 46$ ). 
33 En juillet 1950, le gouvernement péroniste interrompt toutes les expéditions de bœuf vers la Grande-Bretagne ; une suspension qui dure jusqu'en avril 1951. Cependant, la mesure n'améliore pas vraiment le flux de bétail vers le marché intérieur. De façon plus importante, faute d'exportations, la balance commerciale de l'Argentine et son pouvoir d'achat à l'étranger diminuent considérablement. En 1946, les exportations de l'Argentine dépassent les importations de \$ 3969.2 millions m/n. Une telle disparité menace le projet d'industrialisation péroniste. De 1949 à 1952, le volume de machines importées décline de $50 \%$. Cela dit, en 1952 , le montant payé pour ces importations est $60 \%$ plus élevé que trois ans plus tôt ${ }^{40}$. Dans ce contexte, le gouvernement a besoin de reprendre, de maintenir et même d'augmenter la source principale de devises étrangères en Argentine que représentent les exportations de bœuf. La diminution du bétail et les sécheresses récurrentes empêchent la croissance du nombre d'animaux abattus. La seule solution immédiate est la réduction de la consommation nationale de bœuf.

Les justifications aux différentes formes de rationnement alimentaire s'expliquent dans une grande mesure par le contexte. Pendant la Seconde Guerre mondiale, les gouvernements américains et soviétiques font appel au patriotisme et au devoir civique pour mettre en place leur programme de rationnement (Bentley, 1998 : 92-95 ; Borrero in Belasco et Scranton, 2002 : 258-76). Pour sa part, le gouvernement argentin avait imaginé une campagne semblable à une croisade "pour bien manger afin de vivre bien ", capitalisant avec brio sur des connaissances scientifiques, une structure administrative, des croyances populaires et des inquiétudes collectives sur l'alimentation pré-existante ${ }^{41}$. La nouvelle priorité était d'enseigner à la population la distinction entre comer (manger), c'est-à-dire rassasier sa faim, et alimentarse, autrement dit se nourrir pour être en bonne santé. L'administration péroniste n'a jamais rejeté le discours sur le nouveau droit populaire à l'alimentation de luxe abordable. Cependant, cette position est venue contrebalancer ce discours avec deux nouveaux buts importants : la protection de la santé de la population et la préservation de traditions culinaires nationales. Autrefois attentif au plaisir de manger et à l'importance du bœuf dans l'alimentation, le gouvernement s'intéressait désormais à un paradigme alimentaire qui soulignait le bien-être physique, couplé à un régime équilibré et sain, tout en définissant la nourriture non plus comme un luxe mais comme un droit.

Lorsque le gouvernement péroniste fait état de son inquiétude par rapport à la nutrition, son message touche les secteurs progressistes qui luttent sans succès pour promouvoir l'intervention de l'État sur les questions alimentaires (Schleh, 1930 : 293-94 ; Escudero, 1939). Au début des années 1930, Emilio Schleh, médecin et chercheur spécialisé dans l'alimentation, se trouve parmi ceux qui appellent à la création d'un bureau fédéral consacré aux problèmes nutritionnels. Afin de satisfaire cette demande et de bénéficier de la structure institutionnelle et de recherche mise en place sur les vingt années précédentes, Carrillo, le Ministre de la santé, crée le Direction de l'Alimentation. Cette direction inclue l'Institut National de l'Alimentation comme son agence principale. L'institut, d'abord dépendant du gouvernement municipal de Buenos Aires, est nationalisé en 1938 et occupe une position marginale. En tant que directeur, Carrillo nomme Enrique Pierángeli, un disciple et collaborateur proche de Pedro Escudero, fondateur de l'Institut National de l'Alimentation en 1927. Sous l'administration péroniste, l'Institut se concentre sur la recherche alimentaire, la 
diffusion de l'information au public, et la publication de journaux, livres et brochures spécialisés. Il organise également des conférences, des ateliers et des activités éducatives dans les écoles et pour le grand public, tout en conseillant les institutions privées. Le département intervient dans les affaires liées à la production, à la distribution, à la planification et à l'approvisionnement alimentaire au sein des hôpitaux et des écoles publics. Il dirige également la " Police alimentaire » en charge des contrôles sanitaires dans les épiceries, les usines alimentaires et les restaurants.

L'importance croissante de l'alimentation dans les problèmes de l'État se fait encore plus évidente avec le projet de santé publique figurant dans le second plan quinquennal (1952-58). L'alimentation constitue, selon ce plan, un élément central de la médecine sociale préventive : un moyen de parfaire le "capital humain de la nation " (Carrillo, 1975 : 355-66 ; Carrillo, 1975 : 162-64). ${ }^{42}$ En se basant sur cette idée, le gouvernement péroniste tient compte de la recommandation de chercheurs comme Juan Lazarte, un médecin de gauche. En 1938, celui-ci conseille aux autorités nationales de «penser la nourriture comme un problème public et social, et non pas comme un problème à résoudre individuellement", et de s'impliquer par devoir civique dans les questions alimentaires ${ }^{43}$. Les quelques études historiques sur le sujet confirment l'opinion de Lazarte. Elles montrent que les questions alimentaires d'avant le péronisme appartenaient plus au domaine des municipalités qu'à celui des gouvernements nationaux. On constate également qu'elles impliquaient de façon quasi-exclusive le contrôle des prix, ainsi que les subventions et l'approvisionnement alimentaires. Dans certains cas seulement, un tel contrôle impliquait la mise en place de standards alimentaires ainsi que des poursuites judiciaires pour fraude commerciale et falsification. De fait, le gouvernement péroniste a été le premier à faire de l'alimentation un élément central de la politique nationale, un objet d'organisation institutionnelle, un domaine d'expertise et un sujet d'éducation (Arcondo, 2002 ; Remedi, 2003 ; Rock, 1975 : 203-9).

Alors que le lien entre alimentation et santé était un sujet d'intérêt dans la recherche effectuée par les médecins et sociologues depuis les années 1920, il commence vraiment à attirer l'attention du public dans les années 1930. À l'époque, le médecin Pedro Escudero tient une rubrique hebdomadaire à succès dédiée aux sujets comme l'alimentation des ouvriers, la malnutrition, la nourriture et la beauté, dans La Prensa, un journal de grande circulation (Escudero, 1934). À partir des années 1930, les médias popularisent des idées sur le végétarisme, le régime, l'obésité et le jeûne. Quant aux magazines tels que Salud y Cultura, Biocultura, et Vivir!, ils illustrent l'intérêt pour la nourriture naturelle et saine, au moins parmi un lectorat de classe moyenne (Álvarez et Pinotti, 2000 : 77-80). Lorsque dans les années 1950, le gouvernement péroniste lance son appel pour réduire la consommation de bœuf comme solution pour éviter les maladies liées à l'alimentation, il s'adresse à un public habitué à l'idée que "l'on perd plus de vies à table qu'à la guerre $»^{44}$.

Dans sa campagne et lors de débats sur l'alimentation, le gouvernement a bénéficié de l'intérêt de la population qui abandonnait les calories (unités d'énergie) au profit des vitamines, nutriments vitaux aux fonctions corporelles et à la lutte contre les maladies. On avait pris l'habitude d'encenser le bœuf pour son apport de calories mais, dans les années 1950, on commence à considérer les aliments protecteurs comme les principales sources de vitamines (Aguirre, $2004: 18)^{45}$. Contrairement aux décennies précédentes, les publicités alimentaires de ces années-là mentionnent explicitement les types de 
vitamines présentes dans le produit dont il était question, ainsi que leur contribution vitale à la santé du consommateur, et surtout, au bien-être des enfants ${ }^{46}$. La campagne officielle se concentre ainsi sur les bénéfices salutaires d'un régime alimentaire comprenant des quantités proportionnées de différents aliments. Elle privilégie la nutrition à la gourmandise et salue un régime qui symbolise l'innovation, le progrès et la science moderne. L'administration péroniste n'a pas ouvertement remis en question la signification symbolique et matérielle du bœuf pour les Argentins, jusqu'en 1952, mais s'est concentré sur la surconsommation de bœuf. Le gouvernement se montrait franchement opposé au gâchis de nourriture. Et selon Perón : «Pour trois millions de vaches, nous en jetons un million et demi aux ordures $»^{47}$.

Début 1952, la Hotels and Restaurants Confederation, supposément inspirée par la campagne sanitaire officielle, demande au gouvernement d'interdire la vente de bœuf une fois par semaine dans tous les lieux de restauration. Cette demande coïncide étrangement avec la sécheresse récurrente, la réduction du bétail et la pression financière visant à augmenter les exportations : un contexte qui exige des mesures radicales pour réduire la consommation nationale. L'idée de créer l'Association d'hôteliers et restaurateurs pourrait aussi indiquer que la campagne du gouvernement ne produisait pas les résultats escomptés. En février 1952, un décret du gouvernement interdit la vente de bœuf, d'agneau et de porc dans les restaurants le vendredi. Dans le reste du pays, l'interdiction entre en vigueur le jeudi. L'absence de bœuf coïncide avec des mois de pain noir de basse qualité dû au manque de farine, suite à une récolte de blé désastreuse. L'administration proscrit peu après les ventes de bœuf sur les marchés et dans les boucheries, affectant directement la consommation des foyers. L'État intervient dans la sphère de production en interdisant l'abattage de jeunes bêtes femelles (Reca et Gaba, 1973 : 333-46) ${ }^{48}$.

Ainsi, le gouvernement péroniste n'était pas la première institution à considérer la nourriture comme un objet de politique d'État, mais aussi à établir une interdiction légale sur sa consommation. Le gouvernement lève l'interdiction sur l'agneau et le porc en octobre 1952. L'interdiction sur le bœuf se poursuit formellement jusqu'en août 1955, bien que le gouvernement ait cessé de la mettre en pratique en 1953. L'abattage illégal et le marché noir fournissaient quelques moyens d'éviter l'interdiction ; un contrôle gouvernemental strict rend l'achat dans les boucheries et abattoirs illégaux de plus en plus difficile. Ainsi, les gens achètent le bœuf en avance et le stockent dans leur cuisine pour les jours où la vente de viande est proscrite. Cependant, les prix élevés, le manque de stockage réfrigéré convenable et des ventes rationnées ont fait du système de stockage de bœuf une stratégie peu populaire et inadaptée ${ }^{49}$.

41 La campagne gouvernementale s'appuyait sur deux points centraux : convaincre la population que le poisson était une source de satisfaction gustative et un apport en protéines aussi bon que le bœuf, et transformer la consommation de poisson en une icône d'un régime sain et bien équilibré, conforme aux exigences du style de vie de l'époque. Pour soutenir la campagne officielle, le magazine El Hogar affirmait : «C'est un fait qu'alors que la civilisation évolue, la consommation de poisson augmente, elle aussi, graduellement. Et il y a une raison à cela : l'homme civilisé a besoin de phosphate dicalcique, un minéral essentiel au style de vie particulièrement actif d'aujourd'hui $»^{50}$. Le choix des jeudis et vendredis comme jours sans viande renvoyait en effet à la pratique catholique d'éviter le bœuf les jours saints. À Pâques, les gens remplaçaient la viande rouge par le poisson. En capitalisant sur la tradition de manger un aliment en 
particulier, proche de la coutume de manger des pâtes le dimanche, le gouvernement utilisait tous les moyens à sa disposition pour faire du poisson le choix préféré des consommateurs au détriment du bœuf.

La consommation de poisson en Argentine n'avait jamais été très importante en dehors de Pâques. La consommation de poisson de mer se concentrait à Buenos Aires et dans d'autres grosses villes de la région centrale. Quant à celle de poisson de rivière, elle dominait dans le litoral, la région autour de la rivière Paraná. En 1930, la consommation annuelle de poisson par personne à Buenos Aires était de 3,4 kilos. Elle se situait autour d'un kilo dans les terres. Le système de distribution réfrigérée, coûteux et mal organisé, s'appuyait sur l'idée du poisson comme " plat que les pauvres consommaient seulement comme un luxe " depuis des décennies. En 1928, alors que la production totale de viande approchait 290451496 kilos (dont le bœuf, le porc et l'agneau), la production totale de poisson était seulement de 29728917 kilos (Schleh, 1930 : 109, 112-13, 137, $184)^{51}$.

Dès le début des années 1950, le gouvernement se met à contrôler les prix du poisson frais, à surveiller et à réguler les conditions appropriées de stockage et de distribution, et à accorder des crédits généreux pour moderniser les flottes de pêche et les usines de transformation $^{52}$. La production et l'industrialisation $\mathrm{du}$ poisson vivent conséquemment leur plus grande croissance jusqu'alors. Bien qu'en 1946, la production totale de poisson frais, de mer et de rivière, ait été de 58183050 kilos, elle atteignait 77 240900 kilos en 1953. Au fil de cette période, le colin, une espèce typique de la côte argentine, prend la première place dans la production de poisson frais et devient la variété de choix pour les consommateurs ${ }^{53}$. Le développement très important de l'industrie de la conserve de poisson constituait le changement le plus impressionnant mené par la campagne officielle pour encourager sa consommation. Les entreprises de conserves de poisson destinées à la consommation nationale ont connu un essor et sont ainsi devenues la branche la plus moderne et la plus productive de l'industrie alimentaire. En 1953, 150 usines traitent 22663557 kilos de poisson, ce qui équivaut à une production de poisson totale de $29 \%$. Quant au maquereau, il représente plus de $61 \%$ du poisson en conserve. En effet, la croissance de la pêche au maquereau dans les années 1950 répondait à la demande croissante de l'industrie de la conserve, étant donné que ce poisson était presque absent du marché du poisson frais. Alors qu'on ne pêchait pas le maquereau à la fin des années 1930, sa production était de 5390 tonnes en 1946, et elle atteignait 17958 tonnes en 1952 (López, 1955 : 25-33).54

De manière générale, les médias étaient déçus par l'augmentation de la consommation de poisson. Ils avançaient que même avec des événements comme "La semaine du poisson ", une campagne médiatique enthousiaste parrainée par le Ministère de la Santé pour promouvoir la consommation de poisson en septembre 1953, les Argentins continuaient à consommer plus de bœuf ou d'autres viandes que de poisson. En fait, en 1955, l'Argentine était l'un des pays les mieux nourris au monde et se classait parmi les meilleurs en ce qui concerne la consommation de bœuf. Comme les médias populaires l'affirmaient, le poisson ne remplaçait pas le bœuf. Cependant, l'Argentine passe rapidement de la première à la quatrième place parmi les consommateurs de bœuf dans le monde. Et surtout, la consommation de poisson triple, en tout cas à Buenos Aires, un marché qui consommait autour de $54 \%$ de la production totale de poisson. Pendant les premières années du péronisme, la hausse des salaires a joué un rôle important dans l'augmentation de la consommation de bœuf. Dans les dernières années de Perón au 
pouvoir, l'information et l'interdiction légales sur le bœuf ont propulsé la croissance de la consommation de poisson ${ }^{55}$. Une enquête mené à domicilie en 1952 confirme la corrélation entre l'interdiction de bœuf une fois par semaine et l'augmentation de la consommation de poisson. Les enquêtés disaient atteindre $50 \%$ de leur consommation hebdomadaire de poisson le vendredi, jour de l'interdiction de bœuf. Les prix compétitifs du poisson participaient aussi à cette tendance. Même si son prix n'était pas aussi bas que les médias l'exigeaient, le poisson incarnait une alternative économique. En 1953, un kilo de colin coûtait $\$ 2.10 \mathrm{~m} / \mathrm{n}$, alors qu'un kilo de steak revenait à $\$ 7 \mathrm{~m} /$ n. La consommation de poisson par personne à Buenos Aires passe de 3 kilos dans les années 1930 à 9,6 kilos en 1953. Puisque l'interdiction légale de vendre du bœuf le vendredi donnait lieu à une réduction annuelle de la consommation par personne d'environ 5,7 kilos, l'augmentation de la consommation de poisson par personne compensait aisément cette réduction avec une hausse de 4 kilos $^{56}$.

Au-delà des modifications dans les habitudes alimentaires populaires, la campagne d'alimentation péroniste visant à augmenter la consommation de poisson révélait un changement dans l'utilisation et le contenu que le gouvernement faisait du symbolisme alimentaire. Elle soulignait également une transformation dans la diffusion des représentations populaires de l'alimentation. Alors que les médecins avaient prôné la consommation de bœuf comme une source d'énergie, de calories et de fer, ils mettaient désormais en avant le rôle vital des protéines et des minéraux dans un style de vie sain. La presse, qui avait dépeint la consommation de bœuf comme une mesure de bien-être populaire et une source de plaisir, désignait désormais le poisson comme une icône de progrès alimentaire et de civilisation. Alors que dans les statistiques officielles le bœuf était utilisé pour mesurer le pouvoir d'achat des foyers, le poisson devient désormais une nouvelle unité de mesure. Alors que dans un premier temps le gouvernement soutenait que la hausse de salaires améliorerait le régime alimentaire du peuple, par la suite il se concentrerait sur l'information nutritionnelle, l'expertise de spécialistes et l'éducation alimentaire comme sources d'habitudes alimentaires meilleures ${ }^{57}$.

\section{Modernité et tradition alimentaires}

Le gouvernement s'appuyait sur la protection de la santé pour persuader la population de manger moins de bœuf et diversifier son alimentation; il s'agissait également de promouvoir la défense de la tradition. Perón déclare en 1949 :

«Nos criollos installés dans les terres, attirés par les salaires des entreprises industrielles les plus importantes, abandonnent leur bétail, leurs champs et leurs petits vergers pour se rapprocher des centres économiques. Ce changement de lieu de domicile et d'emploi modifie les habitudes alimentaires : en fait, ces hommes remplacent vite locro, mazamorra, humita, achuras grillées et puchero « avec un peu de tout » par les aliments vendus à l'épicerie du coin, par les conserves et les assiettes anglaises $»^{58}$.

Perón contredisait sa déclaration d'avant les élections selon laquelle l'Argentine souffrait de privations, et mystifiait le rôle des secteurs populaires régionaux en tant que réservoirs de la tradition culinaire nationale. Cependant, il synthétisait aussi un développement qui préoccupait le public depuis le début des années 1940 : l'abandon des repas argentins traditionnels. Tout au long des années 1950, les médias se fascinent pour la transformation des habitudes alimentaires populaires, laquelle est considérée comme la conséquence de l'urbanisation, de la migration et de l'industrialisation. 

à l'égard des plats traditionnels argentins désormais perdus. Le magazine Continente demande au gouvernement d'aider les petites entreprises artisanales alimentaires dans la production d'alfeñiques tucumanos, d'alfajores santafesinos, de cordobeses, et d'aguardiente catamarqueña. Ces produits représentaient explicitement le vrai goût argentin tout comme la qualité supérieure des ingrédients locaux de l'artisanat autochtone ${ }^{59}$. En 1949, un spécialiste de l'alimentation venu du nord déplore que «les tables locales aient perdu les plats riches et savoureux qui formaient un témoignage vivant et indiscutable d'un passé d'expériences gustatives paisibles... Ces plats provenaient des secrets de famille, résultats d'une alliance de cuisine indigène traditionnelle et de vieux plats espagnols qui, à force d'échanges de techniques et de saveurs, avaient donné naissance à la cuisine criolla ${ }^{60}$. La presse se lamentait sur le fait que les Argentins ne mangeaient plus de puchero, locro, humita en chala et de quibebe, ou de desserts tels que la mazamorra, le dulce de zapallo, le dulce de leche et l'arroz con leche ${ }^{61}$. Dans les cours de cuisine, les livres, les émissions de radio, les publicités et brochures, le gouvernement proposait les recettes de ces plats régionaux et soulignait l'importance de cuisiner avec des produits locaux, moins chers et disponibles en quantité. Les magazines populaires suivaient l'impulsion du gouvernement en publiant de nombreuses recettes de repas traditionnels, à l'image du célèbre livre de la chef Petrona de Gandulfo. Même l'organe officiel des puissants éleveurs de la province de Santa Fe se plaignait que plus personne ne mange de mazamorra ou de locro, et que les aliments végétariens soient recommandés (Carrizo de Gandulfo, 1951) ${ }^{62}$.

À l'exception du puchero, lequel comporte du bœuf, du porc ou du poulet, les plats mis en avant par le gouvernement péroniste et les médias étaient tous dépourvus de viande. Conformément à l'importance que la campagne gouvernementale donnait à la nourriture on trouvait un grand nombre de plats considérés comme sains et requérant de grandes quantités de légumes frais. En fait, en dehors de ces facteurs (la crise du bétail et le déséquilibre commercial) qui poussaient la campagne à réduire la consommation de bœuf, le régime alimentaire alternatif promu par le gouvernement argentin était hautement nutritif. À l'opposé, dans d'autres exemples historiques comme le régime fasciste en Italie dans les années 1920, l'État a apporté son soutien à des régimes alimentaires inappropriés et nuisibles à la santé de la population, et ce afin de faciliter la balance des paiements (Helstosky, 2006 : 66).

Traditionnellement cuisinés par des femmes, les plats recommandés par le gouvernement péroniste réaffirmaient le rôle essentiel de la femme dans la construction de l'identité culinaire nationale, un mouvement éloigné de la figure de l'asador mâle. Nécessitant une préparation longue et soignée, ces plats célébraient la femme nourricière, protectrice de la force physique de la nation et productrice de plats faits maison. ${ }^{63}$ Rarement servis au restaurant, ces plats constituaient une vraie invitation à rester à la maison. Les recettes exigeaient la combinaison de tous les ingrédients dans la même casserole sur le poêle, une façon économique de cuisiner qui convenait particulièrement aux secteurs populaires ne disposant pas d'une cuisine spacieuse, d'un four et d'une grande variété d'ustensiles de cuisine. De plus, ces plats appartenaient à une tradition culinaire née à l'époque précoloniale et au XIX ${ }^{\text {ème }}$ siècle, et représentaient la cuisine des provinces éloignées, particulièrement des régions nord et centre-ouest. Ainsi, ces préparations pouvaient attirer les migrants de ces zones qui abandonnaient les habitudes culinaires de leur région d'origine. La mise en avant de ces 
recettes par le gouvernement et les médias a peut-être joué un rôle dans la légitimation de cette tradition culinaire qu'autrement les citadins auraient pu associer au sousdéveloppement et à la pauvreté. Les recettes éclipsaient simultanément l'héritage gastronomique de l'immigration européenne, principalement les pâtes et le risotto. En effet, cet héritage a tout d'abord prospéré dans des grosses villes de la Pampa qui sont devenues célèbres pour la nature cosmopolite de leurs plats (Arcondo, 2002:259) ${ }^{64}$.

51 L'administration péroniste et la presse considéraient le terroir du pays comme une source d'inspiration dans la recherche d'une vraie identité culinaire argentine. La popularité de cette idée dans l'imaginaire collectif, tout comme son attrait, sont évidentes dans l'usage fréquent de formules marketing qui se réfèrent à un produit alimentaire comme le "plus criollo» ou comme la représentation du "vrai goût argentin ». Cette tendance est aussi liée aux nombreux rôles importants des gauchos dans les publicités alimentaires, lorsqu'ils sont étrangement associés à des apéritifs importés ou locaux et à du café instantané, improbables remplaçants des produits traditionnels. L'utilisation de ces personnages a peut-être été une stratégie pour vendre des produits « modernes » et « urbains » à un nouveau consommateur, le migrant rural qui vient habiter en ville et qui était représenté dans des publicités, de façon anachronique, comme l'habitant rural traditionnel des terres. En effet, les années 1940 et 1950 ont connu le boom commercial d'une nouvelle marque de biscuits salés du nom de Criollitas, laquelle s'éloignait de la tradition des noms anglais donnés aux biscuits de ce type ${ }^{65}$.

52 La campagne du gouvernement pour promouvoir des huertas de la salud (potagers sains) dans chaque foyer - petites et grandes villes, arrière-cours et balcons - synthétisait plusieurs éléments de la tradition culinaire nationale, que l'on s'imaginait ancrée à la campagne. Le potager symbolisait la vie dans les provinces, la relation étroite des gens à la nature, le travail collaboratif de la famille, la fraicheur de repas chaleureux, le temps et le dévouement dédiés à l'obtention d'ingrédients naturels. Le potager composait une carte postale nostalgique et colorée que l'on adressait au citadin en train de manger seul une boîte de conserve dans une pièce sombre et confinée. C'était aussi une icône d'indépendance alimentaire. Dans un contexte où les légumes étaient chers et rares, le gouvernement avait une bonne raison de promouvoir les potagers personnels. La campagne nationale, inspirée par les victory gardens anglais, cherchait à sensibiliser les enfants dans les écoles grâce à la diffusion d'informations et à la distribution de graines dans les classes. Entre 1949 et 1950, le gouvernement distribue 200000 paquets de graines; on dénombre alors 400 potagers dans les écoles et 5000 potagers personnels (Pierángeli et León López Gil, 1951 : 102-6) ${ }^{66}$.

53 L'appel du gouvernement pour préserver la tradition culinaire nationale renforçait non seulement l'idéal d'un régime alimentaire sain, moins dépendant de la consommation en bœuf, mais redéfinissait aussi le nationalisme qui dominait l'imaginaire alimentaire depuis le début de l'administration péroniste. Avec des arguments prétentieux tels que «La filière argentine de la viande a enseigné au monde civilisé la façon de manger du bœuf », la presse commence à souligner le rôle déterminant du conditionnement de la viande dans les exportations argentines.$^{67}$ En même temps, elle fait abstraction du conflit entre les marchés du bœuf internationaux et intérieurs que le péronisme disait avoir résolu en faveur des consommateurs argentins. Plutôt que d'ancrer le nationalisme dans une position de confrontation envers les marchés étrangers, la défense de la tradition culinaire structure une nouvelle forme de nationalisme 
alimentaire qui combine efficacement patriotisme, préservation de la culture nationale et défense du bien-être populaire. Cependant, de tels intérêts tournent cette fois-ci autour du lien entre la nourriture, l'histoire et la géographie nationales, les produits locaux et les goûts du criollo, ainsi que les techniques culinaires traditionnelles plutôt que de s'intéresser à la connexion entre la nourriture et la souveraineté économique et politique symbolisée par la production et la consommation de bœuf.

Alors que les médias populaires célébraient les habitudes culinaires des provinces et que le gouvernement les promouvaient, les migrants du pays n'exprimaient pas nécessairement leurs préférences et mangeaient de plus en plus grosses quantités de bœuf, particulièrement le churrasco (un steak grillé sans os). La consommation de bœuf dépassait les attentes populaires. Le bœuf était relativement économique, facile et rapide à cuisiner, et détenait une teneur hautement calorique. C'était aussi une excellente source de protéines et il offrait une grande impression de satiété. ${ }^{68}$ Qui plus est, les migrants du pays adoptaient progressivement les habitudes alimentaires citadines d'influence européenne indéniable, et plus spécifiquement porteñas, à l'image du completo (café et lait avec du pain et du beurre ou des croissants), de la pizza, des aliments en conserve et des repas pris à l'extérieur. En effet, au milieu du XX ${ }^{\text {ème }}$ siècle, Buenos Aires devient un "grand magasin gastronomique ». En 1951, la capitale avait quatre fois plus de restaurants qu'en 1945. Plus de 30\% des terrains citadins étaient occupés par des restaurants, et dans des zones comme Leandro Alem Avenue, le taux de concentration était si élevé qu'on dénombrait plus de 25 restaurants en seulement deux quartiers. ${ }^{69}$

En 1947, un décret national demande aux restaurants d'appliquer une réduction de 10\% sur les prix ou d'offrir un "menu économique »- une soupe, deux entrées et un dessert - qui n'excéderait pas le prix fixé par le gouvernement selon le type de restaurant concerné. Le décret établissait aussi qu'en cas d'indisponibilité du plat du jour, les restaurants devraient offrir n'importe quel autre plat figurant sur le menu au prix fixé. ${ }^{70}$ Ainsi, les deux splendides restaurants du Hogar de la Empleada offrait le déjeuner et le dîner à des prix bas et fixes à ceux qui présentaient une preuve d'emploi ou d'affiliation à un syndicat. En 1954, ces restaurants servent des repas à 400000 ouvriers. Le gouvernement encourageait les dirigeants et leur donnait des conseils techniques sur l'ouverture de cantines dans les usines afin d'offrir aux ouvriers des repas sains et au prix abordable (Stawski, $2004: 199)^{71}$.

Même si les gens se rendaient au restaurant principalement pour se distraire, les ouvriers représentaient la part de la population qui mangeait le plus à l'extérieur ; en effet, ils ne disposaient pas d'assez de temps pour rentrer chez eux. Leurs contemporains pensaient que, contrairement aux protocoles rituels requis pour apprécier des repas faits maison, la vie moderne imposait que l'on mange vite. Alors qu'à la fin des années 1930, le copetín al paso - un plat comprenant différents aliments servis ensemble en petites quantités - était inconnu à Buenos Aires, il devient très populaire dans les cafés au milieu des années 1940 . Bon marché et rapide à préparer, il rassemblait les composantes de la notion de "manger à l'ère de la hâte " ${ }^{72}$. Pour les mêmes raisons, les pizzerias ont prospéré pendant ces années-là. Le manque d'assiettes, de tables et de socialisation ont fait de la part de pizza bon marché un aliment moderne, urbain, dépersonnalisé et "démocratique». Contrairement aux États-Unis, où la nourriture industrielle était "démocratique» dans le sens où on la consommait dans toutes les classes sociales, en Argentine, le terme "démocratique » 
faisait uniquement allusion aux classes inférieures et populaires (Boorstin, 1973 : 309-31). Les fractions des classes urbaines moyennes et supérieures associaient la consommation de pizza - qui, depuis le début du XX⿳亠े⿵冂⿰⿱丶㇀⿱㇒丶⿱一土⿵冂丶 dehors de la communauté italienne - aux classes populaires qui vivaient dans les campagnes. Ces même fractions de classe surnommaient péjorativement les migrants de cabecita negra (petite tête noire) ou bien, les « 20 et 20 ", en référence aux 20 cents qu'ils déboursaient pour s'acheter une part de pizza et un verre de bière ou de vin, et les 20 cents supplémentaires pour écouter sur le jukebox de la pizzeria le chanteur populaire d'époque, Antonio Tormo (Gambini, $2001: 28$ ).

En tant qu'élément culinaire de l'identité de la classe ouvrière, la pizza était en compétition avec le completo à Buenos Aires. Le completo, élément classique de l'alimentation des ouvriers, était vendu au café, une autre institution porteña très importante. Pour les ouvriers qui ne pouvaient pas s'offrir de déjeuner ni de dîner au restaurant, le completo remplaçait efficacement un vrai repas en échange de quelques pièces seulement. Au cours de l'été 1948, le journal péroniste La Época lance une campagne pour dénoncer la hausse du prix du completo, avançant qu'il est en train de devenir « le plus gros petit problème du citoyen lambda ». La Época maintient que cette habitude alimentaire porteña est rapidement adoptée par les nouveaux-venus de la ville, mais que la hausse des prix remet en question son attrait populaire. Obligés d'offrir le "déjeuner économique » à un prix fixe, les restaurants et cafés augmentent le coût du completo pour profiter d'un marché captif en pleine expansion ${ }^{73}$.

Manger vite impliquait aussi cuisiner vite, une pratique qu'incarnaient les aliments en conserve et particulièrement les plats tout prêts aussi variés que les raviolis, les spaghettis et le steak à la portugaise. Leurs défenseurs se concentraient sur la qualité élevée, l'hygiène et les économies en temps et en argent que représentaient de tels plats. La nourriture en conserve incarnait l'efficacité de la standardisation moderne grâce aux rations cuisinées qui rendaient le shopping, le stockage et la préparation de la nourriture plus faciles et plus rapides. Sa diversité égayait aussi l'alimentation quotidienne. Selon ce point de vue, grâce à toutes ces caractéristiques, les aliments en conserve assuraient aux consommateurs un niveau de vie élevé, une alimentation variée et la simplification des tâches domestiques. Comme le lavage, la découpe et la cuisson d'ingrédients crus étaient épargnés à la ménagère, particulièrement celle qui travaillait hors de chez elle, elle disposait de plus de temps pour s'occuper d'elle-même, de sa famille et de la communauté ${ }^{74}$.

Cependant, Ramón Carrillo, le Ministre de la santé, conseille aux Argentins de «ne pas se laisser aller aux avantages supposés qu'offrent de tels produits aux ménagères, des avantages qui choisissent pour elles au détriment des aliments frais ; cela nous prépare simplement à la destruction de la mécanique humaine, en ignorant le fait qu'en tant qu'Argentins, nous avons le privilège d'obtenir la quantité nécessaire de produits frais dont nous avons besoin, et ce avec un minimum d'effort ». Les médias populaires décrivaient fréquemment les aliments en conserve comme une tendance américaine répondant à des besoins étrangers tels que l'absence de service domestique, des petites maisons et des petites cuisines, et un style de vie mouvementé. En fait, les Américains avaient un déficit chronique de vitamines et ne connaissaient pas vraiment les plaisirs d'une vraie alimentation. Ces opinions, associées à la campagne nutritionnelle officielle, menaient à l'idéalisation des repas nationaux comme sains, savoureux et 
basés sur des ingrédients frais, et à leur représentation comme des objets de fierté nationale ${ }^{75}$.

Comme l'a très bien exprimé l'anthropologue Sidney Mintz, «lorsque beaucoup d'autres choses changent, les habitudes alimentaires peuvent également évoluer, et de tels changements sont bien souvent imprévisibles » (Mintz, 1996 : 24). Dans l'Argentine $\mathrm{du}$ milieu du $\mathrm{XX}^{\mathrm{ème}}$ siècle, les Argentins contemporains envisageaient ces transformations comme un choc entre deux paradigmes, l'un représentant la modernité - manger à l'extérieur, de la nourriture " rapide " et " démocratique " comme la pizza et les conserves - et l'autre représentant la tradition - les plats végétariens et/ou faits maison ainsi que les recettes régionales, principalement originaires du nord. Cette dichotomie, reflet des inquiétudes collectives suscitées par la migration vers les villes principales et le processus parallèle d'industrialisation, a permis au gouvernement de promouvoir des plats traditionnels et de légitimer sa campagne pour réduire, au nom de la sante publique, la consommation de viande de bœuf.

\section{Conclusions}

61 Toutes les mesures liées à l'approvisionnement en nourriture prises par l'État - les politiques salariales, le contrôle des prix, la régulation de la production et du commerce, les décisions sur les exportations et les importations, le soutien financier à l'industrie agroalimentaire (production et distribution compris), l'organisation institutionnelle, la légalisation et la codification des questions alimentaires et les campagnes nutritionnelles - vont bien au-delà de ce que les gens posent sur leur table. Les politiques alimentaires gouvernementales comprennent la création, l'appropriation et la mobilisation d'un panel de représentations, de discours, de croyances, d'inquiétudes et d'aspirations fortement ancrées dans l'imaginaire. Ces mêmes imaginaires lient les questions alimentaires à des processus politiques, sociaux, culturels et économiques. La politique péroniste ne consiste pas seulement à augmenter la consommation de bœuf par personne : elle redéfinit l'image de l'Argentine au sein de l'économie mondiale, valide le concept d'auto-détermination économique et remet en question le rôle du capitalisme international. De façon tout aussi importante, la politique péroniste dans la filière de la viande de bœuf repositionne les droits des consommateurs nationaux sur le marché externe et, de facto, promeut une idéologie nationaliste, anti-impérialiste et industrialiste pour développer le pays. De plus, une consommation plus élevée de bœuf par personne avait des implications qui dépassaient l'enrichissement en protéines de l'alimentation quotidienne : elle véhiculait une idéologie sur le droit du peuple, marquant la fin du privilège social basé sur un accès inégal à la nourriture. De fait, elle symbolisait parfaitement la conception péroniste de la justice sociale.

62 Appadurai a avancé l'idée selon laquelle la force sémiotique de l'alimentation, impliquant des concepts culturels précis pour son animation et des contextes sociaux spécifiques pour sa mobilisation, demeure tacite. L'État péroniste s'est ainsi approprié, et a appliqué et instrumentalisé efficacement des croyances déjà existantes tout comme des connaissances scientifiques, des aspirations sociales et des inquiétudes collectives-. Dans la culture argentine, le bœuf était un " aliment-totem $»^{76}$. Il constituait une exigence de la classe ouvrière bien ancrée mais jamais satisfaite et incarnait ainsi un 
signe important d'un niveau de vie élevé. Depuis le début du XX ${ }^{\text {ème }}$ siècle, les discours médicaux et populaires considéraient le bœuf comme une source vitale d'énergie et de force. Cependant, le fait que de telles croyances existent déjà ne rendait pas le message moins original. Le gouvernement péroniste apparentait la consommation de bœuf à un style de vie luxueux, signe de l'acquisition d'un statut social plus élevé et un aliment agréable au palais. De la même manière, les inquiétudes exprimées par l'administration péroniste quant à l'abandon des plats traditionnels répondaient aux inquiétudes exprimées dans la presse depuis le début des années 1940 à propos des transformations survenues dans les habitudes alimentaires populaires. Quand le gouvernement a fait de la récupération de la cuisine régionale un aspect central du processus, il s'est approprié de plus belle, tout en les articulant à son avantage, les discours d'alors pour modifier les habitudes alimentaires des argentins ; ces discours abordaient les changements alimentaires liés à l'urbanisation, à l'industrialisation et à la migration. De façon similaire, l'administration péroniste a repris, lors du lancement de sa campagne nutritionnelle, un discours que le corps médical avait établi depuis longtemps, et qui renvoyait l'État à sa responsabilité d'intervenir en faveur de l'éducation alimentaire de la population et d'attirer l'attention des citoyens sur la relation entre l'alimentation et un style de vie sain.

L'intérêt pour la tradition culinaire et une alimentation saine ont remplacé la consommation de bœuf de façon matérielle et symbolique, révélant non seulement la succession de deux paradigmes alimentaires distinctifs basés sur les différentes définitions de "manger correctement "; mais aussi le pouvoir et les intérêts investis de l'État dans la création de ces paradigmes. Jusqu'en 1950, «manger sainement » était synonyme d'abondance, de salaires élevés, d'aliments de luxe, de plaisir et de goût ; c'était une notion qui correspondait parfaitement à la période d'apogée économique, de consolidation politique et d'enthousiasme populaire. Au début des années 1950, lorsque les problèmes de l'industrie du bétail et l'inversion de l'équilibre commercial ont mené à la réduction de la consommation en bœuf, on a ajouté à la définition d'une «alimentation saine » les notions de santé, d'équilibre, de variété, de restriction, d'éducation, d'information, de tradition, d'expression de caution politique croissante, d'organisation institutionnelle et de crise économique.

\section{Secondary Sources}

ABOY Rosa, Viviendas para el pueblo : Espacio urbano y sociabilidad en el barrio Los Perales, 1946-1955, Buenos Aires, Fondo de Cultura Económica, 2005.

AGUILAR-RODRÍGUEZ Sandra, « Cooking Modernity : Nutrition Policies, Class, and Gender in 1940s and 1950s Mexico City », The Americas, vol. 64, n² 2, October 2007, pp. 177-205.

AGUIRRE Patricia, « Toda la carne al asador », Todo es Historia, n 380, March 1999, pp. 80-93. 
AGUIRRE Patricia, Ricos flacos y gordos pobres : La alimentación en crisis, Buenos Aires, Capital Intelectual, 2004.

APPADURAI Arjun, « Gastro-Politics in Hindu South Asia », American Ethnologist, vol. 8, n 3, 1981, pp. 494-511.

ARACONDO Aníbal, Historia de la alimentación en la Argentina : Desde los orígenes hasta 1920, Córdoba, Ferreira Editor, 2002.

BALLENT Anahí, Las huellas de la política : Vivienda, ciudad, peronismo en Buenos Aires, 1943-1955, Buenos Aires, Prometeo, 2005.

BARTHES Roland, Mythologies, New York, Hill and Wang, 1972.

BELASCO Warren and SCRANTON Philip (eds.), Food Nations : Selling Taste in Consumer Societies, New York, Routledge, 2002.

BENTLEY Amy, Eating for Victory : Food Rationing and the Politics of Domesticity, Chicago, University of Illinois Press, 1998.

BIANCHI Susana y SANCHIS Norma, El Partido Peronista Femenino 1946-1955, Buenos Aires, Centro Editor de América Latina, 1988.

BOORSTIN Daniel, The Americas : The Democratic Experience, New York, Random House, 1973.

CORTI Paola, « Emigración y alimentación : Representaciones y autorrepresentaciones en la experiencia de una corriente migratoria regional italiana ", Estudios migratorios latinoamericanos, vol. 12, n 35, 1997, pp. 103-127.

DODDS Klaus, Pink Ice : Britain and the South Atlantic Empire, London, I. B. Tauris, 2002.

DRINOT Paulo, «Food, Race and Working-Class Identity : Restaurantes Populares and Populism in 1930s Peru ", The Americas, vol. 62, n² 2, 2005, pp. 245-70.

ELENA Eduardo, «Justice and Comfort : Peronist Political Culture and the Search for a New Argentina, 1930-1955 », Princeton, Princeton University, PhD dissertation, 2002.

ELENA Eduardo, «Peronist Consumer Politics and the Problem of Domesticating Markets in Argentina, 1943-1955 », Hispanic American Historical Review, vol. 87, n 1, 2007, pp. 111-149.

FERIOLI Néstor, La Fundación Eva Perón, Buenos Aires, Centro Editor de América Latina, 1990.

FERRERAS Norberto, « Evolución de los principales consumos obreros en Buenos Aires, 1880-1920 ", Ciclos 11, n²2, 2001, pp. 157-80.

FIDDES Nick, Meat, A Natural Symbol, London, Routledge, 1991.

FOSTER Nancy and HANDELMAN Howard, "Food Production and Distribution in Cuba" in John C. SUPER and Thomas C. WRIGHT (eds.), Food, Politics, and Society in Latin America, Lincoln, University of Nebraska Press, 1985, pp. 174-98.

GABBACIA Donna R., We Are What We Eat : Ethnic Food and the Making of Americans, Cambridge, Harvard University Press, 1998.

GAMBINI Hugo, Historia del Peronismo, vol. 2, La obsecuencia 1952-1955, Buenos Aires, Planeta, 2001.

GENÉ Marcela, Un mundo feliz: Imágenes de los trabajadores en el primer peronismo, 1946-1955, Buenos Aires, Fondo de Cultura Económica, 2005.

GERCHUNOFF Pablo y Lucas LLACH, El ciclo de la ilusión al desencanto : Un siglo de políticas económicas argentinas, Buenos Aires, Ariel, 1998. 
GERMANI Gino, «El surgimiento del Peronismo : El rol de los obreros y de los migrantes internos ", Desarrollo Económico, vol.13, n 51, 1973, pp. 432-488.

GIRBAL-BLACHA Noemí M., Estado, sociedad y economía y en la Argentina 1930-1997, Buenos Aires, Universidad Nacional de Quilmes, 2001.

HELSTOSKY Carol, Garlic and Oil : Politics and Food in Italy, London, Berg, 2006.

HOROWITZ Roger, Putting Meat on the American Table : Taste, Technology, Transformation, Baltimore, John Hopkins Univ. Press, 2006.

LEVENSTEIN Harvey A., Revolution at the Table : The Transformation of the American Diet, Berkeley, University of California Press, 2003.

MILANESIO Natalia, « The Guardian Angels of the Domestic Economy : Housewives' Responsible Consumption in Peronist Argentina ", Journal of Women's History, vol.18, $\mathrm{n}^{\circ} 3$, September 2006, pp. 91-117.

MINTZ Sidney W., Sweetness and Power : The Place of Sugar in Modern History, New York, Viking, 1985.

MINTZ Sidney W., Tasting Food, Tasting Freedom : Excursions into Eating, Culture, and the Past, Boston, Beacon Press, 1996.

NOVICK Susana, IAPI: Auge y decadencia, Buenos Aires, Centro Editor de América Latina, 1986. OCHOA Enrique C., Feeding Mexico : The Political Uses of Food since 1910, Wilmington, Scholarly Resources, 2000.

PASTORIZA Elisa (ed.), Las puertas al mar : Consumo, ocio y política en Mar del Plata, Montevideo y Viña del Mar, Buenos Aires, Biblos, 2002.

PEFFER E. Louise, « The Argentine Cattle Industry under Perón », Food Research Institute Studies, vol.1, n 2, 1960, pp. 151-184.

PEFFER E. Louise, « State Intervention in the Argentine Meat Packing Industry, Oct. 1, 1946-Dec. 31, 1958 », Food Research Institute Studies, vol. 2, n 1, February 1961, pp. 33-75.

PILCHER Jeffrey M., ; Qué vivan los tamales! Food and the Making of Mexican Identity, Albuquerque, University of New Mexico Press, 1998.

PILCHER Jeffrey M., The Sausage Rebellion : Public Health, Private Enterprise, and Meat in Mexico City, 1890-1917, Albuquerque, University of New Mexico Press, 2006.

PLOTKIN Mariano, Mañana es San Perón : A Cultural History of Perón's Argentina, Wilmington, DE, Scholarly Resources, 2003.

RECA Lucio and Ernesto GABA, « Poder adquisitivo, veda y sustitutos : Un reexamen de la demanda interna de carne vacuna en la Argentina, 1950-1972 », Desarrollo Económico, vol. 13, n 50, 1973, pp. 333-346.

REMEDI Fernando, « Las condiciones de vida material : Cocinas étnicas y consumo alimentario en la provincia de Córdoba a comienzos de siglo » in Marcelo Álvarez y Luisa Virginia Pinotti (eds.), Procesos socioculturales de alimentación, Buenos Aires, Ediciones del Sol, 1997, pp. 99-137.

REMEDI Fernando, Consumo de alimentos, condiciones sanitarias y políticas públicas en la ciudad de Córdoba en las primeras décadas del siglo XX, Córdoba, Editorial Municipalidad de Córdoba, 2003. ROCK David, Politics in Argentina, 1890-1930 : The Rise and Fall of Radicalism, New York, Cambridge University Press, 1975. 
SALVATORE Ricardo, « Heights, Nutrition, and Well-Being in Argentina, ca. 1850-1950 :

Preliminary Results ", Journal of Iberian and Latin American Economic History, vol. 25, n 1, 2007, pp.

53-86.

SCARZANELLA Eugenia, «El ocio peronista : Vacaciones y ‘turismo popular’ en Argentina 19431955 », Entrepasados, vol. 14, 1998, pp. 65-84.

SMITH Peter H., Politics and Beef: Patterns of Conflict and Change, New York, Columbia University Press, 1969.

STAWSKI Martín, « El populismo paralelo : Política social de la Fundación Eva Perón 1946-1955 » in Patricia BERROTARÁN, Aníbal JÁUREGUI y Marcelo ROUGIER (eds.), Sueños de bienestar en la Nueva Argentina : Estado y políticas públicas durante el Peronismo, 1946-1955, Buenos Aires, Imago Mundi, 2004.

SUPER John C, and WRIGHT Thomas C. (eds.), Food, Politics, and Society in Latin America, Lincoln, University of Nebraska Press, 1985.

TOBIN Jeffrey, « Todo mito gauchesco que camina va a parar al asador porteño : El asado y la identidad nacional porteña » in María Cristina PONS, Claudia SORIA ROSARIO y Beatriz Viterbo (eds.), Delirios de grandeza. Los mitos argentinos : Memoria, identidad, cultura, 2005, pp. 211-228.

TORRE Juan Carlos y PASTORIZA Elisa, « La democratización del bienestar » in Juan Carlos TORRE (ed.), Los años peronistas 1943-1955, Buenos Aires, Sudamericana, 1998, pp. 257-312.

WILLIAMS-FORSON Psyche A., Building Houses Out of Chicken Legs : Black Women, Food, and Power, Chapel Hill, University of North Carolina Press, 2006.

\section{Primary Sources}

\section{Periodicals (place of publication is Buenos Aires unless noted)}

Ahora

Aquí Está

Argentina

Caras y Caretas

Claridad

Clarín

Continente

Democracia

Economía y Finanzas

El Hogar

El Laborista

Hechos e Ideas

Investigaciones de Mercado

La Capital (Rosario)

La Época 
La Lucha

La Tribuna (Rosario)

La Prensa

Mucho Gusto

Mundo Peronista

Noticias Gráficas

Nuestras Mujeres

Nuevas Bases

Orientación

Para Tí

Revista de la Asociación Argentina de Dietología

Revista de la Sociedad Rural de Rosario (Rosario)

\section{Government publications and other print primary sources}

CARRILLO Ramón, Organización general del Ministerio de Salud Pública, Buenos Aires, Eudeba, 1975.

CARRILLO Ramón, Planes de Salud Pública 1952-1958, Buenos Aires, Eudeba, 1975.

Fundación Eva Perón.

DE LA TORRE Lisandro, Las carnes argentinas y el monopolio extranjero, Buenos Aires, Colegio Libre de Estudios Superiores, 1947.

Departamento de Investigaciones Pesqueras, Actividades pesqueras en la República Argentina y posibilidades de incrementar el consumo de pescado, Buenos Aires, Ministerio de Agricultura, 1956. Dirección Nacional de Estadística Social, Nivel de vida de la familia obrera: Su evolución durante la Segunda Guerra Mundial, 1939-1945, Buenos Aires, Dirección Nacional de Estadística Social, 1945.

Dirección Nacional del Servicio Estadístico Nacional, Síntesis estadística mensual de la República Argentina, 1946-1955.

DUARTE Erminda, Mi hermana Evita, Buenos Aires, Ediciones Centro de Estudios Eva Perón, 1972.

ESCUDERO Pedro, Alimentación : Colaboraciones publicadas en La Prensa, Buenos Aires, Hachette, 1934.

ESCUDERO Pedro, La política nacional de alimentación en la República Argentina, Buenos Aires, Publicaciones del Instituto Nacional de la Nutrición, vol. 3, 1939.

FERNS H. S., « Britain's Informal Empire in Argentina, 1806-1914 », Past and Present, vol. 4, November 1953, pp. 60-75.

Fundación Eva Perón, Hogares de tránsito, Buenos Aires, Servicio Internacional Publicaciones Argentinas, n.d.

Fundación Eva Perón, El Hogar de la Empleada General San Martín, Buenos Aires, Servicio Internacional Publicaciones Argentinas, n.d.

CARRIZO de GANDULFO Petrona, El libro de Doña Petrona : Recetas de arte culinario, Buenos Aires, Compañía General Fabril Financiera, 1951. 
GONZÁLES ALBERDI, Paulino, Cómo abaratar la vida, Buenos Aires, Anteo, 1942.

GUSTAVINO Roberto, La salud y el consumo de carne, Buenos Aires, Ciencias, 1954.

LICEAGA José, Carnes en la economía argentina, Buenos Aires, Raigal, 1952.

LLORENS Emilio, Encuesta continental sobre el consumo de productos de alimentación, y vestido y sobre la vivienda popular, Montevideo, Consejo Permanente de Asociaciones Americanas de Comercio y Producción, 1944.

Ministerio de Agricultura y Ganadería, Producción pesquera de la República Argentina, Años 1946/53, Buenos Aires, Ministerio de Agricultura y Ganadería, 1955.

Ministerio de Comercio, Informaciones sobre el problema ganadero, Buenos Aires, Instituto Nacional de Carnes, 1956.

Ministerio de Hacienda, Índices del costo del nivel de vida, actividad industrial y costo de la construcción : Informe B. 1., Buenos Aires, Dirección Nacional de Estadísticas y Censos, 1956.

Ministerio de Salud, Libro de la salud, Ministerio de Salud, Buenos Aires, 1952.

PERÓN Juan Domingo, Obras Completas, Buenos Aires, Fundación Hernandarias, 1984.

PIERÁNGELI Enrique, «La subalimentación nacional : El infraconsumo y concepto de alimentos protectores » in Ministerio de Salud Pública de la Nación, Política alimentaria argentina : Conferencias pronunciadas en la campaña de educación sanitaria, Buenos Aires, Departamento de Talleres Gráficos, 1951, pp. 91-103.

SCALABRINI ORTIZ Raúl, Política británica en el Río de la Plata, Buenos Aires, Reconquista, 1940.

SCHLEH Emilio, La alimentación en la Argentina, Buenos Aires, Ferrari, 1930.

SCHLEH Emilio, El subconsumo de alimentos en América del Sur, Buenos Aires, Sudamericana, 1942.

WOODBURY Robert Morse, Food Consumption and Dietary Surveys in the Americas, Montreal, ILO, 1942.

\section{NOTES}

1. "Serán encarcelados los que explotan al pueblo," Ahora, 16 Juillet 1946, couverture.

2. Sur les implications symboliques de l'alimentation Williams-Forson (2006). La collection éditée par Belasco et Scranton (2002), comporte des articles représentatifs des deux écoles universitaires. Les exemples d'études particulièrement centrées sur la politique alimentaire, via l'analyse précise de l'industrie alimentaire et du commerce (Horowitz, 2006 ; Levenstein, 2003 ; Gabaccia, 1998). Parmi les études s'intéressant à la politique alimentaire de l'État voir Pilcher (2006), Ochoa (2000) et Super and Wright (1985).

3. Pour des analyses sur les changements dans les habitudes alimentaires au fil des siècles, voir Jeffrey M. Pilcher (1998) et Sidney W. Mintz (1985).

4. Juan Domingo Perón, "La política alimentaria argentina," Hechos e Ideas, Mai-Juin 1949, p. 353.

5. "Sidra, pan dulce, juguetes y palabras de esperanza llegaron como una bendición," La Época, 28 Dec. 1947, p. 13.

6. "El pueblo argentino vive mal alimentado," Orientación, 8 Oct. 1936, p. 4.

7. "Las ventas minoristas de productos de almacén," Investigaciones de Mercado 1, no. 8 (Juin 1952), p. 2. L'index du coût de la vie (base : $1943=100$ ), particulièrement au niveau de l'alimentation, passa de 144.1 en 1946 à 622.7 en 1952. L'index du coût de la vie se basait sur les dépenses quotidiennes d'un ouvrier non qualifié avec une femme et deux enfants en-dessous de 14 ans, 
domicilés à Buenos Aires. Índices del costo del nivel de vida, actividad industrial y costo de la construcción: Informe B. 1 (Buenos Aires : Ministerio de Hacienda, Dirección Nacional de Estadísticas y Censos, 1956), p. 7.

8. Ramón Carrillo, "El criterio biológico en el reordenamiento económico de la alimentación en la Argentina," Hechos e Ideas, Nov. 1951, p. 276.

9. "Que cada habitante pueda comer 150 gramos más de carne: He ahí la solución a la falta de mercados," Orientación, 7 Juillet 1940, p. 4.

10. "Las tratativas sobre carnes con Gran Bretaña," El Laborista, 25 Mai 1949, p. 1.

11. Avec sa position nationaliste, le gouvernement péroniste montra aussi sa détermination à récupérer les îles Malvinas, alors sous occupation britannique, et revendiqua une partie de la péninsule antarctique (Dodds, $2002: 37-51$ ).

12. "De afuera vendrá quien lo tuyo se llevará," Caras y Caretas, Août 1952, pp. 54-55.

13. "Argentina el mayor consumidor de carne vacuna pero el menor de carne ovina y porcina," Economía y Finanzas, 14 Avr. 1955, pp. 211-19. Sur cette période, le déclin des exportations était une tendance économique générale relativement prononcée pour les grains.

14. Le gaucho est l'habitant nomade typique des pampas, connu pour ses talents d'éleveur. Les pampas sont les plaines qui s'étendent sur la province de Buenos Aires, Santa Fe, La Pampa, et Córdoba.

15. Escudero, Alimentación, p. 79.

16. Sur l'asado et la masculinité aujourd'hui, voir Aguirre (1999: 83) et Tobin (2005:211-28). Amy Bentley (1998: 87-89), universitaire spécialisée dans les questions alimentaires, souligne l'identification entre viande et masculinité aux États-Unis.

17. "Consumo aparente de carne y pescado en la Capital Federal en los últimos 20 años", Investigaciones de Mercado, 7 Mai 1954. En 1956, des statistiques publiées par l'Institut National de la filière des viandes font état de différents chiffres mais démontrent toujours l'augmentation de la consommation de bœuf par habitant en Argentine : de 76,6 kilos en 1935 à 93 kilos en 1950. Ministerio de Comercio, Instituto Nacional de Carnes, Informaciones sobre el problema ganadero, Buenos Aires, 1956, p. 122.

18. Malheureusement, l'administration péroniste n'entreprit pas d'études complètes ou ne compila pas de séries de statistiques fiables sur la consommation alimentaire après 1946.

19. Perón, "La política alimentaria argentina," p. 362.

20. En fait, au tournant du $20^{\text {ème }}$ siècle, la consommation annuelle de bœuf en Italie du sud était de 6,4 kilos (Remedi, 1997 : 99-137, 124 ; Corti, 1997 : 103-27, 111).

21. González Alberdi, Cómo abaratar la vida, p. 27 ; "La solución del problema de las carnes es impedida por el trust," Orientación, 18 Juillet 1940, p. 4 ; “Coma más carne," Orientación, 31 Oct. 1940 , p. 8.

22. "En ninguna parte hay tantos alimentos y tan baratos como en la Argentina," Ahora, 20 Nov. 1947, pp. 14-15.

23. Pour une évaluation du bien-être biologique pendant la première partie du $20^{\text {ème }}$ siècle basée sur des estimations de taille relevées sur les registres de prisonniers et recrues militaires, voir Salvatore (2007: 53-86).

24. "Las comidas de nuestros abuelos," Aquí Está, 13 Jan. 1947, pp. 14-15.

25. "Consumo de carnes en la Argentina," Investigaciones de Mercado, 10 Mai 1956.

26. J'ai adapté cette expression du terme "famine de viande" de Nick Fiddes (1991 : 14).

27. Dirección Nacional de Estadística Social, Nivel de vida de la familia obrera: Su evolución durante la Segunda Guerra Mundial, 1939-1945 (Buenos Aires, 1945), p. 84.

28. Escudero, Alimentación, p. 252.

29. Chaque année, le gouvernement et la Fondation Eva Perón distribuaient des millions de bouteilles de cidre et de pan dulce marqués des visages des Perón, et ce dans tout le pays. De plus, il y eut des ventes record de produits alimentaires et de boissons de Noël dans ces années-là. "Las 
Navidades de Perón y Evita," Mundo Peronista, 1 Jan. 1954, pp. 34-39 ; "En ningún año se han comido tantos pavos y pan dulces," Ahora, 20 Dec. 1949, p. 19 ; "Las horcas caudinas del pan dulce y la sidra," La Lucha, 20 Sept. 1949, p. 6.

30. Scarzanella, "El ocio peronista," p. 65-84.

31. À propos du Hogar de la Empleada, voir Ferioli (1990 : 106-7) et Elena (2002 : 194-203).

32. Fundación Eva Perón, Hogares de tránsito (Buenos Aires : Servicio Internacional Publicaciones Argentinas, no date), n. p.

33. Subsecretaría de Informaciones, Por la República y por ella (Buenos Aires : n.p., n.d.)..

34. "La teoría alimentaria del General Perón," Nuevas Bases, 15 Dec. 1952, p. 1 ; "Política alimentaria," Nuestras Mujeres, 1er Juin 1949, p. 11.

35. Germinal Rodríguez, "Los alimentos dentro del presupuesto obrero y su relación con los salarios," dans Ministerio de Salud Pública de la Nación, Política alimentaria argentina, 59-90, p. 71.

36. Carrillo, "El criterio biológico en el reordenamiento económico de la alimentación en la Argentina," pp. 280-81 ; José Alloati, "Hacia un sistema de distribución racional de alimentos en todo el país," dans Ministerio de Salud Pública de la Nación, Política alimentaria argentina, pp. 14875.

37. "Inaugúrase el Congreso de Racionalización Alimentaria," El Laborista, 24 Nov. 1952, p. 5.

38. “¿ Sabe comer bien el pueblo argentino ? ” El Hogar, 11 Jan. 1952, p. 3-4 ; "El médico en casa: la alimentación," Para Tí, 5 Mar. 1929, p. 89.

39. La sécheresse provoqua aussi une perte majeure dans les récoltes et une réduction consécutive du volume de grains tant pour la consommation nationale que pour l'exportation.

40. Dirección Nacional del Servicio Estadístico Nacional, Síntesis estadística mensual de la República Argentina, Jan. 1948, no. 1, p. 47, en juillet 1954, no. 7, pp. 813, 842.

41. Ministerio de Salud, Libro de la salud (Buenos Aires, 1952), pp. 49-50.

42. "El mejor de los capitales es la salud del pueblo," Mundo Peronista, 15 Jan. 1954, pp. 19-20.

43. Juan Lazarte, "La alimentación y el pueblo", Claridad, Mai 1938, pp. 10-12.

44. "El menú de los viernes sin carne," El Hogar, 4 Avr. 1952, p. 12.

45. "Consigna para el hogar: Comer bien pero sin derrochar," Democracia, 10 Juin 1952, p. 3 ; "Ya no somos los mayores consumidores de carne," Democracia, 5 Sept. 1955, p. 3.

46. Condibé Ad, Para Tí, 14 May 1946 ; Risko Ad, Para Ti, 11 Nov. 1946.

47. Juan Perón, "El consumo y la especulación," Hechos e Ideas, Août 1950, 420-21 ; “Cocina de la salud: Enseñaremos a no matar los alimentos," Mucho Gusto, Dec. 1950, pp. 20-21.

48. "Prescindirán de carne un día por semana las casas de comida de toda la república," Clarín, 31 Jan. 1952, pp. 1-2. L'interdiction fut réaffirmée en 1964-65 (deux fois par semaine) et en 1971.

49. "La provision de alimentos," La Capital, 24 Fev. 1952, p. 4 ; "La dieta del pueblo argentino," La Tribuna, 2 Fev. 1952, p. 6.

50. “ $¿$ Es la pesca una gran riqueza nacional ?” El Hogar, 11 Avr. 1952, p. 3 ; “Sin carne, pero los menús fueron abundantes," Democracia, 23 Fev. 1952, p. 3.

51. "Consumiéronse 178500 kilos de pescado," Clarín, 23 Fev. 1952, p. 3.

52. "El fomento pesquero," Mundo Peronista, 15 Jan. 1955, p. 31.

53. "Ministerio de Agricultura y Ganadería, Producción pesquera de la República Argentina, Años 1946/53 (Buenos Aires, 1955), 11, p. 453 ; “En el 83,4\% de los casos, las amas de casa de la Capital Federal que consumen pescado fresco, prefieren merluza," Investigaciones de Mercado, Sept. 1952, pp. 6-10.

54. Ministerio de Agricultura y Ganadería, Producción Pesquera de la República Argentina, Años 1946/53, 501 ; “Más pescado y menos carne," La Prensa, 13 Sept. 1953, p. 4.

55. "Ya no somos los mayores consumidores de carne," Democracia, 5 Sept. 1955 ; "El pescado es sano y barato," Caras y Caretas, Oct. 1953, pp. 123-24.

56. Reca et Gaba, "Poder adquisitivo, veda y sustitutos", p. 336. "Consumo aparente de carne y pescado en la Capital Federal en los últimos 20 años," Investigaciones de Mercado, Mai 1954, p. 7. "El 
consumo de pescado de mar y mariscos en la Capital Federal," Investigaciones de Mercado, Jan. 1955, p. 10 ; Departamento de Investigaciones Pesqueras, Actividades pesqueras en la República Argentina y posibilidades de incrementar el consumo de pescado (Buenos Aires: Ministerio de Agricultura, 1956), pp. 31-32.

57. "Un tema muy argentino: La carne y el pescado," La Prensa, 22 Oct. 1954, p. 3 ; "El pescado es sano y barato", Caras y Caretas, Oct. 1953, pp. 123-24.

58. Perón, "La política alimentaria argentina," p. 355. Criollo renvoie aux habitants des provinces nord et nord-ouest de l'Argentine. Le locro est un ragoût préparé avec du maïs, des haricots et de la citrouille. La mazamorra est un gateau sucré, laiteux et composé de maïs, semblable au pudding. La humita est composé de maïs et la humita en chala est servie dans des feuilles de maïs. Les achuras sont des abats. Le puchero est un ragoût de porc, de bœuf ou de poulet auquel on ajoute du maïs, des pommes de terre, de la citrouille et de l'oignon.

59. “Alfeñiques, alfajores, empanadas, etc.," Continente, 15 Oct. 1947, p. 174. Les alfeñiques sont des bonbons très sucrés originaires de la province de Tucumán et préparés avec du sucre de canne. Les alfajores sont des petits gâteaux collés les uns aux autres par du dulce de leche, un caramel marron préparé en faisant chauffer du lait sucré ou de la confiture de fruits. Santa Fe et Córdoba sont connus pour leurs différents styles d'alfajores. L'aguardiente est une boisson alcoolisée dérivée du sucre de canne et produite dans la province de Catamarca.

60. Orestes Di Lullo, “ $i$ El pueblo no sabe comer ?” Argentina, Oct. 1949, p. 61.

61. Le quibebe se prépare avec de la citrouille écrasée et du fromage. Le dulce de zapallo est une gelée à la citrouille. L'arroz con leche est un gâteau sucré préparé avec du lait et du riz.

62. “¿Falta comida o carecemos de la capacidad doméstica para utilizar los elementos disponibles que permiten amplia y suculenta alimentación ?" Revista de la Sociedad Rural de Rosario, Sept.-Oct. 1954, pp. 96-97 ; “El menú para los viernes sin carne,” El Hogar, 25 Apr. 1952, p. 18 ; Jaime Espejo Sola, "Errores de alimentación típicamente argentinos," dans Ministerio de Salud Pública de la Nación, Política alimentaria argentina, pp. 253-56.

63. Sur les questions de genre et le rôle des femmes pendant le péronisme après 1950, voir Milanesio (2006 : 91-117).

64. "Las comidas de nuestros abuelos," Aquí Está, Jan. 13, 1947, pp. 14-15.

65. Nescafé Ad, Para Ti, 30 July 1946, p. 31 ; Fernet Branca Ad, Mundo Peronista, 15 Oct. 1951, quatrième de couverture ; Cocinero Ad, Ahora, 31 Août 1946, p. 5 ; Fernando Rocchi, "De las masitas para el té a la galletita peronista," La Maga, 3 Jan. 1996, p. 39.

66. "El huerto familiar restablecerá el equilibrio en la demanda de productos de consumo," El Laborista, 30 Mar. 1952, p. 2.

67. "El bife criollo: Victoria de nuestros frigoríficos," Noticias Gráficas, 27 Nov. 1950, p. 10.

68. Horacio Storni, "La dieta ideal argentina y los factores de la alimentación popular," dans Ministerio de Salud Pública de la Nación, Política alimentaria argentina, p. 117.

69. "Buenos Aires de hoy," Continente, Dec. 1951, p. 91 ; "Veinticinco restaurantes y hoteles en dos manzanas," Caras y Caretas, Mar. 1952, pp. 132-34.

70. “Se establecieron categorías y precios de un menú económico," La Argentina, 25 Jan. 1947, p. 3.

71. Fundación Eva Perón, El Hogar de la Empleada General San Martín (Buenos Aires : Servicio Internacional Publicaciones Argentinas, n. d.).

72. "La estadística nos dice que comemos demasiado," Mundo Argentino, 7 Fev. 1945, pp. 3-5 ; "Las comidas de nuestros abuelos," Aquí Está, 13 Jan. 1947, pp. 4-15 ; "Hay que salvar las vitaminas!" Aquí Está, 3 Nov. 1947, pp. 2-3.

73. "La Época inicia la campaña pro café con leche barato," La Época, 22 Jan. 1948, p. 5 ; “El completo debe ser despachado a bajo precio," La Época, 1 Feb. 1948, p. 12 ; "Reivindicación de la pizza," Continente, 15 Juillet 1948, p. 40 ; “Origen y función social de la pizzería," Argentina, Août 1947, pp. 34-35. 
74. "La industria de las conservas ha dado al país nuevas costumbres culinarias," La Época, 20 Oct. 1955, p. 4 ; “Alimentos envasados : Síntesis del progreso de una industria y posibilidad popular de comer mejor", El Laborista, 23 Mar. 1950, p. 4 ; Swift Ads, El Hogar, 4 Nov. 1949 et 14 Juillet 1950, quatrième de couverture.

Carrillo, "El criterio biológico en el reordenamiento económico de la alimentación en la Argentina", p. 269.

75. "Gracias al uso de alimentos frescos," Continente, 15 Nov. 1947, pp. 72-73.

76. Roland Barthes (1972: 58) utilisait "boisson-totem" pour affirmer l'importance du vin dans la culture française.

\section{RÉSUMÉS}

Dès les débuts du gouvernement de Juan Domingo Perón, les pratiques alimentaires constituent non seulement une dimension essentielle de la politique de l'État, mais aussi un élément central de la propagande gouvernementale. Cet article s'oppose à la séparation analytique de l'étude des politiques de celles des imaginaires afin d'ouvrir de nouvelles pistes de réflexion sur le péronisme. Dans un premier temps, nous mettons l'accent sur le rôle central joué par les dimensions économiques, politiques et iconographiques de l'alimentation dans les politiques publiques, dans la culture commerciale, la santé publique, ainsi que dans les définitions du bienêtre, qu'il soit social, national ou physique. Dans un deuxième temps, en replaçant l'étude de l'alimentation dans une nouvelle perspective, nous réinterprétons les éléments clés de l'idéologie "péroniste» que sont le nationalisme et le programme social argentin mis en place à cette période. Au-delà de son rôle comme symbole du bien-être des classes populaires, l'augmentation de la consommation de bœuf par tête a ébranlé l'image de l'Argentine comme économie exportatrice asservit au capitalisme international. En privilégiant la consommation intérieure au détriment des marchés extérieurs, la politique péroniste concernant la filière bovine a créé une puissante idéologie cherchant à atteindre la souveraineté économique. Cette idéologie a permis à l'État de renforcer son engagement dans la distribution de la richesse nationale au profit de la population locale. Le gouvernement a déployé une série de mesures entre 1946 et 1949 afin d'augmenter la consommation de bœuf parmi la classe ouvrière pour laquelle cette denrée était considérée comme un luxe. Notre analyse démontre enfin que le Péronisme n'a cessé de s'approprier et d'instrumentaliser les principales controverses en Argentine pour redéfinir la culture populaire, les discours d'inspiration de gauche et le discours médical, concernant notamment la consommation de bœuf et le nationalisme, le luxe, le droit et la santé, ainsi que l'intervention de l'État dans le domaine nutritionnel, l'éducation alimentaire du peuple et le lien entre le nationalisme, la tradition et la culture culinaire.

From the beginning of Juan Domingo Perón's administration, food consumption was both a significant object of state policy and a central component of official propaganda. This essay resists the analytical separation between politics and imaginaries in order to expand our understanding of Peronism in new directions. First, it shows the economic, political, and iconographic centrality of food for state planning, commercial culture, public health, and definitions of social, national, and physical wellbeing. Second, the essay reinterprets nationalism and social entitlement-concepts that researchers have identified as key in Peronist ideologythrough a new focus on food. An increase in per-capita beef consumption-beyond serving as a 
symbol of popular wellbeing-undermined the images of Argentina as an export economy subservient to foreign capitalism. By favoring internal consumers over external markets, Peronist beef politics created an empowering ideology of economic sovereignty. This ideology reinforced the commitment of the state to benefit the local population in the distribution of national wealth. Between 1946 and 1949, the government popularized the rise in beef intake as the new entitlement of the working classes to what had previously been a "luxury food". Finally, the analysis demonstrates that Peronism collected and instrumentally continued or redefined key arguments circulating in Argentine popular culture and medical and leftist discourses including the relation between beef and nationalism, luxury, right, and health ; the intervention of the state in nutritional issues; the dietary education of the masses; and the connection between nationalism, tradition, and culinary culture.

Desde el inicio del gobierno peronista encarnado por Juan Domingo Perón, las prácticas alimenticias constituyen no solamente un punto central de la política de Estado en Argentina, sino también un componente fundamental de la propaganda gubernamental. Alejándonos de la visión que separa lo político de lo imaginario, este artículo busca abrir nuevas pistas de reflexión sobre el peronismo. En primer lugar, nos concentraremos sobre el papel de las dimensiones económicas, políticas e icnográficas de la alimentación en la definición de las políticas públicas, la cultura comercial, la salud pública, y el bienestar social, físico y más ampliamente, nacional. En segundo lugar, estudiando la alimentación bajo una nueva óptica interpretamos los elementos clave de la ideología "peronista", es decir el nacionalismo y el programa social puesto en marcha durante esta época. Más allá de simbolizar el bienestar de las clases populares, el aumento per capita del consumo de carne bovina desmitifica la imagen de economía exportadora de la Argentina y muestra su avasallamiento al capitalismo foráneo. Privilegiando a los consumidores internos, en detrimento de los mercados internacionales, la política peronista concerniente a la rama de carne bovina creó una poderosa ideología que buscaba alcanzar la soberanía económica. Dicha ideología permitió al Estado reforzar su compromiso distribuyendo la riqueza nacional en beneficio de la población local. El gobierno desplegó una serie de medidas entre 1946 y 1949 con el fin de aumentar el consumo interno de carne bovina, hasta entonces un lujo para la clase obrera. Nuestro análisis demuestra en última instancia que el Peronismo nunca dejó de apropiarse y de instrumentalizar las principales controversias en Argentina para redefinir la cultura popular, el discurso de izquierda, el discurso médico, principalmente la relación entre el consumo de carne bovina y el nacionalismo, el lujo, el derecho y la salud, así como la intervención del Estado en el área de la nutrición, la educación alimentaria del pueblo y el vínculo entre el nacionalismo, la tradición y la cultura culinaria.

\section{INDEX}

Mots-clés : Péronisme, politique alimentaire, nationalisme, bœuf, imaginaires alimentaires.

Palabras claves : Peronismo, política alimentaria, nacionalismo, carne bovina, imaginarios alimentarios.

Keywords : Peronism, Food politics, Nationalism, Beef, Food imaginaries

\section{AUTEUR}

\section{NATALIA MILANESIO}

Natalia Milanesio is assistant professor of History at the University of Houston. She has published articles on modern Argentine history in the Hispanic American Historical Review, Gender and History, 
the Journal of Women's History, and the Journal of Social History. Her book Workers Go Shopping in Argentina: The Rise of Popular Consumer Culture (University of New Mexico Press) is forthcoming in 2013. Email : nmilane2atcentralpointuhpointedu 